\title{
Estimated Prey Consumption by Harp seals (Phoca groenlandica), Hooded seals (Cystophora cristata), Grey seals (Halichoerus grypus) and Harbour seals (Phoca vitulina) in Atlantic Canada
}

\author{
M. O. Hammill \\ Maurice Lamontagne Institute, P. O. Box 1000 \\ Mont Joli, Quebec, Canada Q5H 3R4 \\ and \\ G. B. Stenson \\ Northwest Atlantic Fisheries Centre, P. O. Box 5667 \\ St John's, Newfoundland, Canada A1C 5X1
}

\begin{abstract}
Consumption of prey by harp (Phoca groenlandica), hooded (Cystophora cristata), grey (Halichoerus grypus), and harbour (Phoca vitulina) seals in Atlantic Canada was estimated for the period 1990-96 by synthesizing and integrating information on individual energy requirements, population size, distribution and diet composition. Total annual consumption by these four species increased from 3.1 million to 4.0 million tons over the seven-year period. Seventy-seven percent (by weight) of the total prey consumption consisted of fish, of which capelin and sand lance were the dominant species accounting for $49 \%$ (by weight) of the total fish consumed. The majority (74\%) of total prey consumption occurred off southern Labrador and Newfoundland (Div. 2J and 3KL), followed by the northern Gulf of St Lawrence (Div. 4RS) (18\%), and the eastern Scotian Shelf (Div. 4VsW) (4\%). Harp seals were the most important predator, accounting for $82 \%$ of total consumption, followed by hooded seals (10\% of total prey consumption), grey seals $(7.8 \%)$, and harbour seals $(0.2 \%)$. Regional differences existed in predation impacts of the four pinnipeds; harp seals were most important in Div. 2J and 3KL and in Div. 4RS, hooded seals were most important in Div. 2J and 3KL and Div. 3M (Flemish Cap), while grey seal predation predominated in the southern Gulf of St. Lawrence (Div. 4T) and on the Scotian Shelf (Div. $4 \mathrm{VsW}$ ). Of the 3.1 million tons of fish consumed by the four species of seals in 1996 , only about $20 \%$ accounted for commercial species such as Greenland halibut (7\%), Atlantic cod (6\%), redfish (4\%), and Atlantic herring (3\%). Most of the consumption of these commercial species consisted of juveniles.
\end{abstract}

Key words: feeding/food, grey seals, harbour seals, harp seals, hooded seals, Northwest Atlantic Ocean

\section{Introduction}

The failure in the Canadian east coast groundfish fishery has been attributed to a combination of excess harvesting, uncertain biological assessments and ineffective fisheries management (FRCC, 1999). Although fishery closures were enacted in the early-1990s, few groundfish stocks have shown signs of significant recovery (FRCC, 1999).

Like elsewhere in the world, fish stocks in Canada have traditionally been managed on a single species basis, although it has long been recognized that a multispecies approach would be more appropriate (Mercer, 1982). Multispecies models offer an improvement over the single species approach because they tend to explicitly account for predatorprey relationships among species (Sparre, 1991). Unfortunately, these models are extremely data intensive (Walters et al., 1997), and often ignore lower trophic levels, giving a very incomplete picture of overall energy flows (Christensen and Pauly, 1993; Christensen, 1995). 
Over the last two decades, seal populations in Atlantic Canada have increased markedly (Mohn and Bowen, 1996; Hammill et al., 1997; Stenson et al., 1997a). Marine mammals, because of their large size and abundance, are thought to have an important influence on the structure and function of many marine ecosystems (Bowen, 1997). However, little empirical evidence exists on the ecosystem impact of marine mammals. One obvious impact is that large quantities of prey are consumed by marine mammals. Evaluating the magnitude of this consumption requires information on population size, energetic requirements, diet composition, sizeclasses and energy density of the prey, as well as the distribution of marine mammal feeding effort (Harwood and Croxall, 1988; Harwood, 1992).

Four species of seals are common in the waters of Atlantic Canada. Grey seals (Halichoerus grypus) and harbour seals (Phoca vitulina) are abundant year-round along the coast from the northeastern United States to Hamilton Inlet and in the Gulf of St Lawrence (Mansfield, 1967), while harp seals (Phoca groenlandica) and hooded seals (Cystophora cristata) are seasonal residents (Sergeant, 1976). In 1994, harp seals were estimated to have consumed 88000 tons (95\% CI: $46000-$ 140000 tons) of Atlantic cod off the Labrador and east coast of Newfoundland, and 54000 tons of cod (95\% CI: 14 000-102 000 tons) in the Gulf of St Lawrence (Stenson et al., 1997a). In 1993, grey seals were estimated to have consumed 39000 tons of cod off the Atlantic coast of Nova Scotia and in the Gulf of St Lawrence (Hammill and Mohn, MS 1994). Hammill et al. (1997) estimated that during 1991-95 hooded seals annually consumed between 63 and 100 tons of Atlantic cod in the Gulf of St Lawrence.

The principal objective of this study was to estimate prey consumption by seals in Atlantic Canada. In this study, estimates of total prey consumption are derived for harp, hooded, grey and harbour seals in Northwest Atlantic waters off southern Labrador and Newfoundland (NAFO Div. $2 \mathrm{~J}$ and $3 \mathrm{KL}$ ), in the northern Gulf of St. Lawrence (Div. 4RS), the southern Gulf of St. Lawrence (Div. 4T), the eastern Scotian Shelf (Div. 4VsW), the Flemish Cap (Div. 3M), and in 'Other' waters of Atlantic Canada (Bay of Fundy: Div. 4X; southern coast of Newfoundland: Subdiv. 3Ps). The relative importance of predation by each species in each area is identified and evaluated.

\section{Materials and Methods}

Estimates of prey consumption were developed by modeling changes in population size, energy requirements, diet composition and seasonal changes in distribution for each of the four pinniped species (Appendix 1).

\section{Population Dynamics}

Age-specific estimates of harp seal abundance were obtained using the approach of Shelton et al. (1996), projected forward to 1996 taking into account available harvest data. Shelton et al. (1996) modeled the dynamics of harp seals using two formulations: one in which pup mortality was assumed equal to adult mortality, and another in which pup mortality was equal to three times the adult mortality rate. Mammals typically have a 'U'-shaped mortality curve with high mortality rates at birth that decline as maturity is attained but which increase in old age (Caughley, 1977). Therefore, the second formulation of the model was used. The model was also updated by incorporating revised catch statistics from the Greenland harvest (Stenson et al., MS 1999).

For grey, hooded and harbour seals, the dynamics of these populations were reconstructed by modifying estimates of survivorship and age-specific reproductive rates to produce a transition matrix (Leslie, 1945) which generated the observed population changes. To determine the stable age composition, the models were initiated with a starting population of 500 pups and the populations 'grown' until there were no changes in the proportional age composition. For hooded and grey seals, total population abundance estimates are lacking; only data on pup production are available. Hence, total population sizes were derived by dividing the pup production in each species by the fraction of pups expected in a population having the modeled stable age structure. The number of animals in each age-class was then determined by multiplying the total population by the proportion of animals in each age-class from the population model. A similar approach was used for harbour seals by allowing a population of 500 pups to grow until no further changes occurred in the proportional age composition. Total population size was then multiplied by the proportion age composition in the 'stable' population to derive the population age distribution.

In all cases, first-year pup mortality was assumed to be greater than mortality in older (age 1+) 
seals. The mortality rate of older seals was assumed to be constant across years and cohorts. Mortality rates of males and females were assumed to be identical, and the sex ratio at birth was assumed to be $1: 1$.

\section{Energy Requirements}

Energy requirements were assumed to be constant throughout the year. Age-specific energy requirements were calculated using:

$$
G E I_{i}=G P_{i} *\left(A F * 293 * B M_{i}^{0.75}\right) / M E
$$

where $G E I_{i}$ is daily gross energy intake (kjoules/ day) at age $i$, and GP is the additional energy required by young seals (< age 6). $G P_{i}$ was set at 1.8 , $1.6,1.4,1.3,1.1,1.1$, and 1.0 for animals aged 0 , $1,2,3,4,5$, and $\geq 6$ yrs respectively (Olesiuk, 1993). The activity factor (AF) was assumed to be 2 (Worthy, 1990) to approximate the average daily energy requirements as a multiple of the basal metabolic rate $\left(293 * B M_{\mathrm{i}}^{0.75}\right.$; Kleiber 1975$)$ where $B M_{i}$ is body mass-at-age in $\mathrm{kg}$. The metabolizable energy (ME) was set at 0.83 (Ronald et al., 1984) based on the assumption that seals primarily consume fish. Growth in body mass-at-age $i\left(B M_{i}\right)$ was modeled using a re-parameterized form of the Gompertz growth curve (Hammill et al., 1995):

$$
B M_{i}=W_{\infty}\left(\frac{W_{0}}{W_{\infty}}\right)^{\exp }\left[\frac{k_{0} \cdot i}{W_{0} \ln \left(W_{0} / W_{\infty}\right)}\right]
$$

where body mass $\left(B M_{i}\right)$, asymptotic weight $W_{\infty}$, and weight at birth $\left(W_{0}\right)$ are in $\mathrm{kg}, i$ is age (in years) and $k_{0}$ is the rate of growth at birth (Table 1). Parameters of the growth curves for each species were determined using Proc NLIN (SAS Institute, 1987).

\section{Seasonal Distribution}

The study region off eastern Canada was divided into six areas based upon NAFO scientific and statistical Divisions and Subdivisions. These areas included: eastern Newfoundland and southern Labrador (Div. 2J and 3KL); the northern Gulf of St Lawrence (Div. 4RS); the southern Gulf of St Lawrence (Div. 4T); the eastern Scotian Shelf (Div. $4 \mathrm{VsW}$ ); the Flemish Cap (Div. 3M) and 'Other' used primarily for grey and harbour seals in the Bay of Fundy (Div. 4X) and along the southern coast Newfoundland (Subdiv. 3Ps) (Fig. 1). The seasonal distribution of each species in each of these areas was estimated from field observations, tag returns, aerial survey observations, historical catch data, anecdotal reports and satellite telemetry.

\section{Diet Composition}

The proportion of each prey species in the diet was determined by reconstructing the wet weight of prey ingested from stomach and/or faeces samples. Where possible, diet composition was analyzed by season and location. No correction was made for unidentified fish. In deriving annual consumption estimates, diet compositions were assumed to remain constant throughout the 1990-96

TABLE 1. Parameters used in the Gompertz growth curves to estimate body size in harp, hooded, grey and harbour

\begin{tabular}{|c|c|c|c|c|c|}
\hline Species/sex & $\mathrm{N}$ & $\begin{array}{l}\text { Asymtotic weight } \\
(\mathrm{W} \infty)(\mathrm{kg})\end{array}$ & $\begin{array}{l}\text { Weight at } t_{\mathrm{o}} \\
\left(\mathrm{W}_{0}\right)(\mathrm{kg})\end{array}$ & $\begin{array}{r}\text { Growth rate } \\
\left(\mathrm{k}_{0}\right)(\mathrm{kg} / \mathrm{y})\end{array}$ & Source \\
\hline Harp seal-male & 967 & 102.60 & 34.20 & 11.27 & Chabot et al. (1996) \\
\hline Harp seal-female & 800 & 98.60 & 30.80 & 12.30 & Chabot et al. (1996) \\
\hline Hooded seal-male & 289 & 256.66 & 36.79 & 30.71 & Stenson (unpubl.) \\
\hline Hooded seal-female & 744 & 187.07 & 38.22 & 19.54 & Stenson (unpubl.) \\
\hline Grey seal-male & 95 & 250.44 & 48.58 & 19.50 & Hammill (unpubl.) \\
\hline Grey seal-female & 166 & 188.21 & 53.92 & 12.54 & Hammill (unpubl.) \\
\hline Harbour seal-male & 70 & 93.13 & 22.62 & 10.51 & Lesage (unpubl.) ${ }^{1}$ \\
\hline Harbour seal-female & 53 & 74.91 & 19.52 & 10.35 & Lesage (unpubl.) ${ }^{1}$ \\
\hline
\end{tabular}
seals. $\mathrm{N}=$ Number of individuals measured.

\footnotetext{
V. Lesage, Maurice-Lamontagne Institute, Mont-Joli, Quebec, Canada.
} 


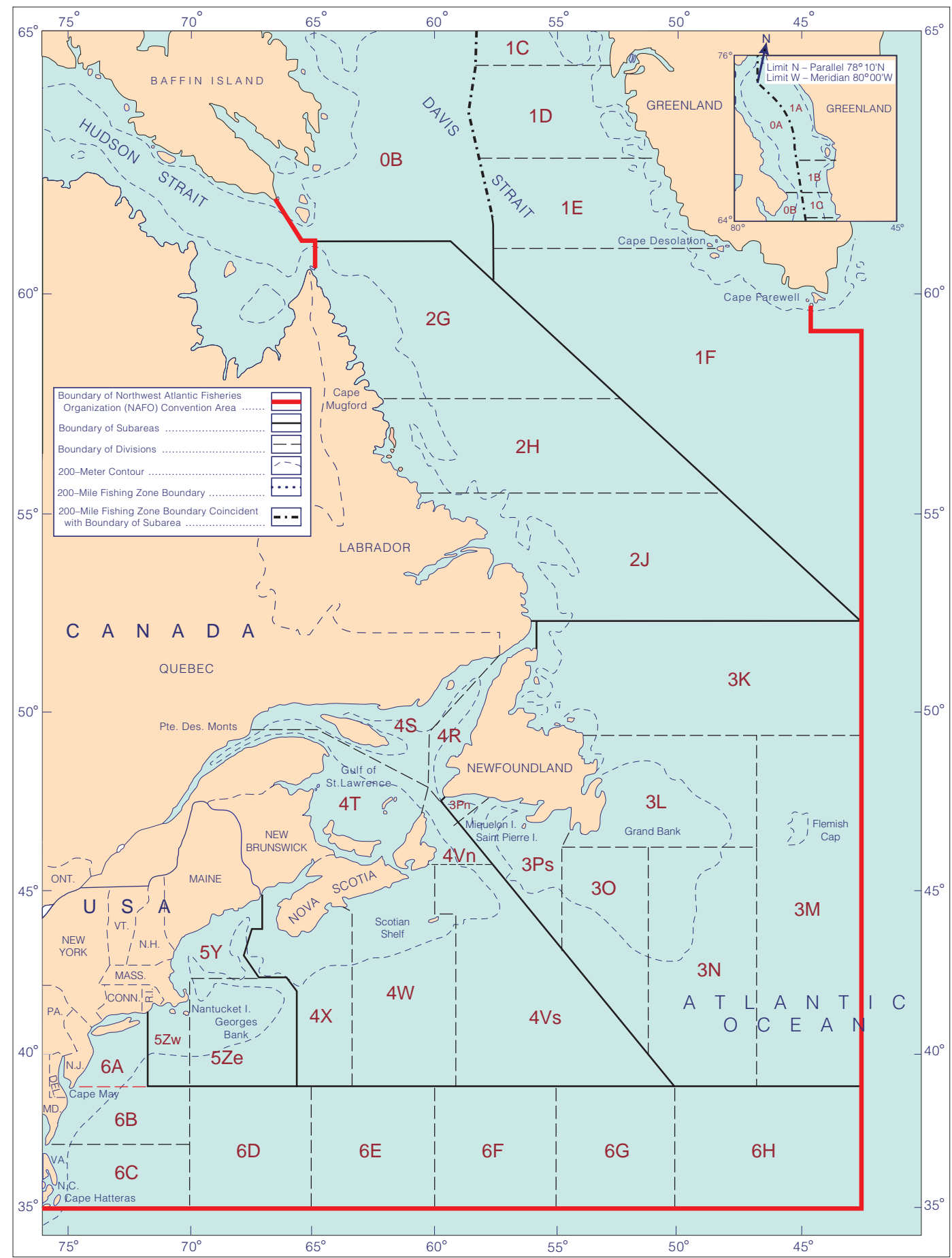

Fig. 1. Northwest Atlantic Fisheries Organization (NAFO) Scientific and Statistical Divisions and Subdivisions. Area designations used in the seal study include: eastern Newfoundland and southern Labrador (Div. 2J3KL); northern Gulf of St. Lawrence (Div. 4RS); southern Gulf of St. Lawrence (Div. 4T); eastern Nova Scotian Shelf (Div. 4VsW); Flemish Cap (Div. 3M); and 'Other' [Bay of Fundy: Div. 4X; southern coast of Newfoundland: Subdiv. 3Ps]. 
period. The mean energy density of prey items in each area/season was estimated using a weighted average of published energy values for individual prey species (Croxall and Prince, 1982; Griffiths, 1977; Hislop et al., 1991; Hodder et al., 1973; Tyler, 1973; Percy and Fife, 1981; Montevecchi and Piatt, 1984; Steimle and Terranova, 1984; Proust, 1996; Martensson et al., 1996; Lawson et al., 1995b, 1998b; Chabot, Institute Maurice Lamontagne, Department of Fisheries and Oceans, Quebec, unpubl. data).

\section{Prey Size}

The size composition of Atlantic cod (Gadus morhua), Atlantic herring (Clupea harengus), redfish (Sebastes spp.) and Greenland halibut (Reinhardtius Hippoglossoides) consumed by seals was estimated using size frequency data from seal dietary studies (harp seals: Lawson and Stenson, 1997; hooded seals: Ross, MS 1993; grey seals: Benoit and Bowen, 1990; Proust, 1996; harbour seals: Bowen and Harrison, 1996).

\section{Results}

Changes in population size have been routinely monitored in harp, hooded and grey seals (Bowen et al., 1987; Stobo and Zwanenberg, 1990; Zwanenberg and Bowen, 1990; Shelton et al., 1997; Stenson et al., 1997b; Hammill et al., 1998; Stenson et al., MS 1999). The abundance of harbour seals, however, has never been adequately censused in Atlantic Canada. Harp seals are the most abundant pinnipeds, with a total population size in eastern Canada estimated to be 4.2 million in 1990, increasing to 5.2 million animals in 1996 (Stenson et al., MS 1999). Hooded seals increased from 470000 animals in 1990 to about 627000 in 1996, while grey seals increased from approximately 97000 animals in 1990 to 173000 animals in 1996. Harbour seal abundance increased from about 23000 animals in 1990 to 32000 in 1996 (Table 2).

Total estimated prey consumption for the four seal species combined increased from 3.1 million tons in 1990 to 4.0 million tons in 1996 (Table 3). Most of this consumption (74\%) occurred off eastern Newfoundland and southern Labrador (Div. 2J and $3 \mathrm{KL}$ ), followed by the northern Gulf of $\mathrm{St}$ Lawrence (Div. 4RS) (18\%), the eastern Scotian Shelf (Div. 4VsW) (4\%), the southern Gulf of St Lawrence (Div. 4T) (1\%), and the Flemish Cap (Div. $3 \mathrm{M})(0.9 \%)$ (Table 4$)$. Consumption of fish accounted for $77 \%$ by weight of the total prey consumed by seals (Table 5). Fish accounted for $72 \%$ of the total prey consumed by seals in Div. $2 \mathrm{~J}$ and $3 \mathrm{KL}$, and for between $87 \%$ and $97 \%$ in the other areas (Table 5). Three prey items accounted for over half $(51 \%)$ of the total consumption (Table 5): capelin (Mallotus villosus) (26\%), shrimp (Pandalus spp.) (13\%) and sand lance (Ammodytes spp.) (12\%). However, the relative importance of forage species differed among areas. In Div. 2J and $3 \mathrm{KL}$, capelin, shrimp and sand lance accounted for $24 \%$, $17 \%$ and $12 \%$, respectively, of the total prey consumed by seals. In the northern Gulf of St Lawrence (Div. 4RS), the most important prey items were capelin (45\%), redfish (Sebastes spp.) (13\%) and pleuronectid flounders (10\%). On the Flemish Cap (Div. 3M), Greenland halibut (Reinhardtius hippoglossoides) (32\%), witch flounder (Glyptocephalus cynoglossus) (16\%), squid (13\%) and Atlantic cod (Gadus morhua) (10\%) were the principal prey items, while in the eastern Scotian Shelf (Div. 4VsW) and in the southern Gulf of St. Lawrence (Div. 4T), sand lance and Atlantic cod were the major forage species.

TABLE 2. Estimated population abundance (number of animals) of harp, hooded, grey and harbour seals in Atlantic Canada between 1990-96, and annual net rates of population increase.

\begin{tabular}{lccrc}
\hline \hline Year & $\begin{array}{c}\text { Harp seal } \\
(3.87 \% / y r)\end{array}$ & $\begin{array}{c}\text { Hooded seal } \\
(4.8 \% / \mathrm{yr})\end{array}$ & $\begin{array}{c}\text { Grey seal } \\
(9.7 \% / \mathrm{yr})\end{array}$ & $\begin{array}{c}\text { Harbour seal } \\
(5.6 \% / \mathrm{yr})\end{array}$ \\
\hline 1990 & 4193200 & 469900 & 96900 & 22800 \\
1991 & 4355000 & 493000 & 106600 & 24100 \\
1992 & 4556200 & 517200 & 117400 & 25500 \\
1993 & 4694200 & 542700 & 129000 & 27000 \\
1994 & 4915800 & 569400 & 142400 & 28500 \\
1995 & 5075600 & 597300 & 156100 & 30100 \\
1996 & 5236800 & 626700 & 173500 & 31900 \\
\hline
\end{tabular}


During the 1990-96 period, seal consumption of Greenland halibut increased from 159000 tons to 207000 tons; Atlantic cod from 132000 tons to 184000 tons; redfish from 105000 tons to 134500 tons; and Atlantic herring from 60000 tons to 84000 tons (Table 3). However, together these commercial species accounted for only about $20 \%$ of the total fish consumption by seals and only $15 \%$ of the aggregate prey consumption.

Harp seals were the most important pinniped predator (Tables 6 and 7) accounting for $78 \%$ of the total consumption of fish by seals and $82 \%$ of the overall prey consumption, followed by hooded

TABLE 3. Estimated prey consumption (tons) by harp, hooded, grey and harbour seals in Atlantic Canada, 1990-96.

\begin{tabular}{|c|c|c|c|c|c|c|c|}
\hline \multirow[b]{2}{*}{ Prey Species } & \multicolumn{7}{|c|}{ Year } \\
\hline & 1990 & 1991 & 1992 & 1993 & 1994 & 1995 & 1996 \\
\hline Capelin (Mallotus villosus) & 825560 & 866841 & 894200 & 924671 & 968402 & 1001626 & 1037741 \\
\hline Sand lance (Ammodytes sp.) & 352640 & 371153 & 392630 & 411984 & 437481 & 459436 & 484764 \\
\hline Pleuronectidae & 211743 & 222973 & 231051 & 240167 & 252380 & 262489 & 273548 \\
\hline Greenland halibut (Reinhardtius hippglossoides) & 158737 & 165878 & 173812 & 181266 & 190037 & 198197 & 206895 \\
\hline Atlantic cod (Gadus morhua) & 132426 & 140858 & 147349 & 155258 & 164753 & 173731 & 183740 \\
\hline Arctic cod (Boreogadus saida) & 146104 & 151665 & 158514 & 163630 & 171306 & 177115 & 183049 \\
\hline Redfish (Sebastes sp.) & 105279 & 112491 & 114041 & 118552 & 124266 & 129175 & 134489 \\
\hline Atlantic herring (Clupea harengus) & 60320 & 64108 & 67147 & 70704 & 75093 & 79177 & 83688 \\
\hline Witch flounder (Glyptocephalus cynoglossus) & 42540 & 44632 & 46826 & 49129 & 51545 & 54079 & 56738 \\
\hline Grenadier (Macrouridae) & 19557 & 20732 & 21980 & 23330 & 24781 & 26325 & 28005 \\
\hline American Plaice (Hippoglossoides platessoides) & 13260 & 13798 & 14451 & 14967 & 15710 & 16290 & 16905 \\
\hline Sculpin (Myxocephalus sp.) & 12931 & 13597 & 14016 & 14506 & 15200 & 15742 & 16321 \\
\hline Blue hake (Antimora rostrata) & 11524 & 12090 & 12684 & 13308 & 13962 & 14648 & 15368 \\
\hline Mackerel (Scomber scombrus) & 8842 & 9607 & 10410 & 11340 & 12369 & 13445 & 14706 \\
\hline Silver hake (Merluccius bilinearis) & 8076 & 8889 & 9580 & 10462 & 11468 & 12543 & 13761 \\
\hline Windowpane (Scopthalmus aquosus) & 10654 & 11029 & 11480 & 11915 & 12478 & 12949 & 13458 \\
\hline Gadus sp. & 5902 & 6204 & 6349 & 6554 & 6843 & 7094 & 7356 \\
\hline Lumpfish (Cyclopterus lumpus) & 3812 & 4111 & 4417 & 4775 & 5168 & 5568 & 6044 \\
\hline Lancetfish (Alepisaurus sp.) & 4390 & 4606 & 4832 & 5070 & 5319 & 5580 & 5855 \\
\hline Wolffish (Anarhichas sp.) & 3754 & 4029 & 4306 & 4632 & 4988 & 5343 & 5770 \\
\hline White hake (Urophycis tenuis) & 4363 & 4549 & 4742 & 4905 & 5141 & 5317 & 5512 \\
\hline Pollock (Pollachius virens) & 2313 & 2538 & 2785 & 3062 & 3371 & 3709 & 4091 \\
\hline Haddock (Melanogrammus aeglefinus) & 2766 & 2979 & 3009 & 3140 & 3304 & 3446 & 3605 \\
\hline Atlantic salmon (Salmo salar) & 2467 & 2652 & 2688 & 2807 & 2955 & 3084 & 3229 \\
\hline Ocean pout (Macrozoarces americanus) & 1649 & 1770 & 1891 & 2033 & 2189 & 2345 & 2531 \\
\hline Eelpout (Lycodes sp.) & 1139 & 1196 & 1256 & 1319 & 1385 & 1454 & 1528 \\
\hline Yellowtail flounder (Pleuronectes ferrugineus) & 956 & 1027 & 1098 & 1182 & 1274 & 1365 & 1475 \\
\hline Alewife (Alosa pseudoharengus) & 687 & 735 & 779 & 832 & 891 & 948 & 1019 \\
\hline Skates (Raja sp.) & 554 & 595 & 637 & 686 & 739 & 793 & 857 \\
\hline Rainbow smelt (Osmerus mordax) & 189 & 200 & 204 & 212 & 222 & 230 & 238 \\
\hline Winter flounder (Pleuronectes americanus) & 110 & 117 & 125 & 133 & 142 & 151 & 162 \\
\hline Cunner (Tautogolabrus adspersus) & 52 & 56 & 59 & 64 & 68 & 73 & 78 \\
\hline Other Fish & 225327 & 234624 & 244421 & 252558 & 264405 & 273650 & 283132 \\
\hline Total Fish & 2380623 & 2502329 & 2603769 & 2709153 & 2849635 & 2967117 & 3095658 \\
\hline Shrimp & 421680 & 437879 & 457158 & 471688 & 493761 & 510215 & 527202 \\
\hline Squid & 56823 & 59963 & 62937 & 66186 & 69842 & 73510 & 77442 \\
\hline Euphausiid & 4761 & 4943 & 5166 & 5331 & 5582 & 5768 & 5957 \\
\hline Other Invertebrates & 239262 & 249449 & 259317 & 267755 & 280324 & 289602 & 299585 \\
\hline Total Invertebrates & 722526 & 752234 & 784578 & 810960 & 849509 & 879095 & 910186 \\
\hline Total Prey & 3103149 & 3254563 & 3388347 & 3520113 & 3699144 & 3846212 & 4005844 \\
\hline
\end{tabular}


TABLE 4. Estimated total prey consumption (tons) by harp, hooded, grey and harbour seals in Atlantic Canada in 1996, by NAFO area and prey species (see species list in Table 3).

\begin{tabular}{|c|c|c|c|c|c|c|c|}
\hline \multirow[b]{2}{*}{ Prey Species } & \multicolumn{7}{|c|}{ NAFO Area } \\
\hline & $2 \mathrm{~J} 3 \mathrm{KL}$ & $4 \mathrm{RS}$ & $3 \mathrm{M}$ & $4 \mathrm{VsW}$ & $4 \mathrm{~T}$ & Other & Total \\
\hline Capelin & 711736 & 323539 & 332 & 179 & 826 & 1129 & 1037741 \\
\hline Sand lance & 345429 & 6182 & 0 & 88892 & 18662 & 25599 & 484764 \\
\hline Pleuronectidae & 179224 & 72706 & 3067 & 12626 & 2498 & 3427 & 273548 \\
\hline Greenland halibut & 191270 & 3913 & 11677 & 0 & 15 & 20 & 206895 \\
\hline Atlantic cod & 82494 & 56099 & 3732 & 24260 & 7235 & 9920 & 183740 \\
\hline Arctic cod & 180763 & 2286 & 0 & 0 & 0 & 0 & 183049 \\
\hline Redfish & 34505 & 97002 & 1219 & 1164 & 252 & 347 & 134489 \\
\hline Atlantic herring & 35845 & 27816 & 0 & 12953 & 2989 & 4085 & 83688 \\
\hline Witch flounder & 50989 & 0 & 5727 & 0 & 9 & 13 & 56738 \\
\hline Grenadier & 18751 & 0 & 2106 & 4241 & 1225 & 1682 & 28005 \\
\hline American plaice & 15141 & 1214 & 0 & 0 & 232 & 318 & 16905 \\
\hline Sculpin & 10431 & 5723 & 0 & 7 & 68 & 92 & 16321 \\
\hline Blue hake & 13816 & 0 & 1552 & 0 & 0 & 0 & 15368 \\
\hline Mackerel & 7 & 5396 & 0 & 5038 & 1799 & 2466 & 14706 \\
\hline Silver hake & 3 & 3047 & 0 & 7678 & 1279 & 1754 & 13761 \\
\hline Windowpane & 0 & 13458 & 0 & 0 & 0 & 0 & 13458 \\
\hline Gadus sp. & 3902 & 3155 & 0 & 224 & 33 & 42 & 7356 \\
\hline Lumpfish & 0 & 2646 & 0 & 0 & 1433 & 1965 & 6044 \\
\hline Lancetfish & 5263 & 0 & 591 & 0 & 0 & 1 & 5855 \\
\hline Wolffish & 0 & 3934 & 0 & 0 & 774 & 1062 & 5770 \\
\hline White hake & 4713 & 624 & 0 & 94 & 35 & 46 & 5512 \\
\hline Pollock & 60 & 222 & 0 & 2767 & 443 & 599 & 4091 \\
\hline Haddock & 0 & 3421 & 0 & 3 & 76 & 105 & 3605 \\
\hline Atlantic salmon & 103 & 2993 & 0 & 0 & 56 & 77 & 3229 \\
\hline Ocean pout & 4 & 1688 & 0 & 26 & 343 & 470 & 2531 \\
\hline Eelpout & 1316 & 42 & 148 & 0 & 9 & 13 & 1528 \\
\hline Yellowtail flounder & 0 & 967 & 0 & 0 & 214 & 294 & 1475 \\
\hline Alewife & 9 & 771 & 0 & 55 & 79 & 105 & 1019 \\
\hline Skates & 0 & 539 & 0 & 0 & 134 & 184 & 857 \\
\hline Smelt & 102 & 135 & 0 & 0 & 1 & 0 & 238 \\
\hline Winter flounder & 6 & 69 & 0 & 42 & 19 & 26 & 162 \\
\hline Cunner & 1 & 45 & 0 & 10 & 9 & 13 & 78 \\
\hline Other Fish & 258267 & 22326 & 2143 & 297 & 44 & 55 & 283132 \\
\hline Total Fish & 2144150 & 661958 & 32294 & 160556 & 40791 & 55909 & 3095658 \\
\hline Shrimp & 513456 & 13541 & 0 & 154 & 23 & 28 & 527202 \\
\hline Squid & 55270 & 7367 & 4656 & 7034 & 1317 & 1798 & 77442 \\
\hline Euphausiids & 5891 & 66 & 0 & 0 & 0 & 0 & 5957 \\
\hline Other Invertebrates & 262009 & 37548 & 0 & 21 & 3 & 4 & 299585 \\
\hline Total Invertebrates & 836626 & 58522 & 4656 & 7209 & 1343 & 1830 & 910186 \\
\hline Total Prey & 2980776 & 720480 & 36950 & 167765 & 42134 & 57739 & 4005844 \\
\hline$\%$ of Annual Total & $74.4 \%$ & $18.0 \%$ & $0.9 \%$ & $4.2 \%$ & $1.1 \%$ & $1.4 \%$ & \\
\hline
\end{tabular}

seals (12\% fish; $10 \%$ total) and grey seals $(10 \%$ fish; $8 \%$ total). Harbour seals accounted for an insignificant percentage $(0.2 \%)$ of the total prey consumption.
The predation impacts of each of the four seal species differed by area (Tables 8 and 9). Off eastern Newfoundland and Labrador (Div. 2J and 3KL) where both harp and hooded seals are abundant, 
TABLE 5. Estimated total prey consumption (\% by weight) by harp, hooded, grey and harbour seals in Atlantic Canada in 1996, by NAFO area and prey species (see species list in Table 3).

\begin{tabular}{|c|c|c|c|c|c|c|c|}
\hline \multirow[b]{2}{*}{ Prey Species } & \multicolumn{7}{|c|}{ NAFO Area } \\
\hline & $2 \mathrm{~J} 3 \mathrm{KL}$ & $4 \mathrm{RS}$ & $3 \mathrm{M}$ & $4 \mathrm{VsW}$ & $4 \mathrm{~T}$ & Other & All Areas \\
\hline Capelin & 23.9 & 44.9 & 0.9 & 0.1 & 2.0 & 2.0 & 25.9 \\
\hline Sand lance & 11.6 & 0.9 & 0.0 & 53.0 & 44.3 & 44.3 & 12.1 \\
\hline Pleuronectidae & 6.0 & 10.1 & 8.3 & 7.5 & 5.9 & 5.9 & 6.8 \\
\hline Greenland halibut & 6.4 & 0.5 & 31.6 & 0.0 & 0.0 & 0.0 & 5.2 \\
\hline Atlantic cod & 2.8 & 7.8 & 10.1 & 14.5 & 17.2 & 17.2 & 4.6 \\
\hline Arctic cod & 6.1 & 0.3 & 0.0 & 0.0 & 0.0 & 0.0 & 4.6 \\
\hline Redfish & 1.2 & 13.5 & 3.3 & 0.7 & 0.6 & 0.6 & 3.4 \\
\hline Atlantic herring & 1.2 & 3.9 & 0.0 & 7.7 & 7.1 & 7.1 & 2.1 \\
\hline Witch flounder & 1.7 & 0.0 & 15.5 & 0.0 & 0.0 & 0.0 & 1.4 \\
\hline Grenadier & 0.6 & 0.0 & 5.7 & 2.5 & 2.9 & 2.9 & 0.7 \\
\hline American plaice & 0.5 & 0.2 & 0.0 & 0.0 & 0.6 & 0.6 & 0.4 \\
\hline Sculpin & 0.3 & 0.8 & 0.0 & 0.0 & 0.2 & 0.2 & 0.4 \\
\hline Blue hake & 0.5 & 0.0 & 4.2 & 0.0 & 0.0 & 0.0 & 0.4 \\
\hline Mackerel & 0.0 & 0.7 & 0.0 & 3.0 & 4.3 & 4.3 & 0.4 \\
\hline Silver hake & 0.0 & 0.4 & 0.0 & 4.6 & 3.0 & 3.0 & 0.3 \\
\hline Windowpane & 0.0 & 1.9 & 0.0 & 0.0 & 0.0 & 0.0 & 0.3 \\
\hline Gadus sp. & 0.1 & 0.4 & 0.0 & 0.1 & 0.1 & 0.1 & 0.2 \\
\hline Lumpfish & 0.0 & 0.4 & 0.0 & 0.0 & 3.4 & 3.4 & 0.2 \\
\hline Lancetfish & 0.2 & 0.0 & 1.6 & 0.0 & 0.0 & 0.0 & 0.1 \\
\hline Wolffish & 0.0 & 0.5 & 0.0 & 0.0 & 1.8 & 1.8 & 0.1 \\
\hline White hake & 0.2 & 0.1 & 0.0 & 0.1 & 0.1 & 0.1 & 0.1 \\
\hline Pollock & 0.0 & 0.0 & 0.0 & 1.6 & 1.1 & 1.0 & 0.1 \\
\hline Haddock & 0.0 & 0.5 & 0.0 & 0.0 & 0.2 & 0.2 & 0.1 \\
\hline Atlantic salmon & 0.0 & 0.4 & 0.0 & 0.0 & 0.1 & 0.1 & 0.1 \\
\hline Ocean pout & 0.0 & 0.2 & 0.0 & 0.0 & 0.8 & 0.8 & 0.1 \\
\hline Eelpout & 0.0 & 0.0 & 0.4 & 0.0 & 0.0 & 0.0 & 0.0 \\
\hline Yellowtail flounder & 0.0 & 0.1 & 0.0 & 0.0 & 0.5 & 0.5 & 0.0 \\
\hline Alewife & 0.0 & 0.1 & 0.0 & 0.0 & 0.2 & 0.2 & 0.0 \\
\hline Skates & 0.0 & 0.1 & 0.0 & 0.0 & 0.3 & 0.3 & 0.0 \\
\hline Smelt & 0.0 & 0.0 & 0.0 & 0.0 & 0.0 & 0.0 & 0.0 \\
\hline Winter flounder & 0.0 & 0.0 & 0.0 & 0.0 & 0.0 & 0.0 & 0.0 \\
\hline Cunner & 0.0 & 0.0 & 0.0 & 0.0 & 0.0 & 0.0 & 0.0 \\
\hline Other Fish & 8.7 & 3.1 & 5.8 & 0.2 & 0.1 & 0.1 & 7.1 \\
\hline Total Fish & 71.9 & 91.9 & 87.4 & 95.7 & 96.8 & 96.8 & 77.3 \\
\hline Shrimp & 17.2 & 1.9 & 0.0 & 0.1 & 0.1 & 0.0 & 13.2 \\
\hline Squid & 1.9 & 1.0 & 12.6 & 4.2 & 3.1 & 3.1 & 1.9 \\
\hline Euphausiids & 0.2 & 0.0 & 0.0 & 0.0 & 0.0 & 0.0 & 0.1 \\
\hline Other Invertebrates & 8.8 & 5.2 & 0.0 & 0.0 & 0.0 & 0.0 & 7.5 \\
\hline Total Invertebrates & 28.1 & 8.1 & 12.6 & 4.3 & 3.2 & 3.2 & 22.7 \\
\hline Total Prey & 100.0 & 100.0 & 100.0 & 100.0 & 100.0 & 100.0 & 100.0 \\
\hline
\end{tabular}

harp seals were the most important pinniped predator accounting for $88 \%$ of the total prey consumed by seals in this area, $59 \%$ of the Atlantic cod consumed, $86 \%$ of the capelin consumed, and more than
$97 \%$ of the pinniped consumption of Arctic cod (Boreogadus saida) and capelin (Table 9). Hooded seals were responsible for most of the pinniped consumption of redfish $(52 \%)$ and Greenland halibut 
TABLE 6. Estimated prey consumption (tons) in Atlantic Canada in 1996, by NAFO area and major prey type, for four species of seals.

\begin{tabular}{|c|c|c|c|c|c|c|c|c|}
\hline \multirow[b]{2}{*}{ Seal Species } & & \multicolumn{6}{|c|}{ NAFO Area } & \multirow[b]{2}{*}{ All Areas } \\
\hline & & $2 \mathrm{~J} 3 \mathrm{KL}$ & $4 \mathrm{RS}$ & $3 \mathrm{M}$ & $4 \mathrm{VsW}$ & $4 \mathrm{~T}$ & Other & \\
\hline \multirow[t]{3}{*}{ Harp Seal } & Fish Prey & 1824791 & 602173 & 0 & 0 & 0 & 0 & 2426964 \\
\hline & Invertebrate Prey & 792638 & 57526 & 0 & 0 & 0 & 0 & 850164 \\
\hline & Total Prey & 2617429 & 659699 & 0 & 0 & 0 & 0 & 3277128 \\
\hline \multirow[t]{3}{*}{ Hooded Seal } & Fish Prey & 319008 & 8297 & 32294 & 0 & 0 & 0 & 359599 \\
\hline & Invertebrate Prey & 43894 & 643 & 4656 & 0 & 0 & 0 & 49193 \\
\hline & Total Prey & 362902 & 8940 & 36950 & 0 & 0 & 0 & 408792 \\
\hline \multirow[t]{3}{*}{ Grey Seal } & Fish Prey & 0 & 50185 & 0 & 158135 & 40430 & 55460 & 304210 \\
\hline & Invertebrate Prey & 0 & 0 & 0 & 6553 & 1245 & 1709 & 9507 \\
\hline & Total Prey & 0 & 50185 & 0 & 164688 & 41675 & 57169 & 313717 \\
\hline \multirow[t]{3}{*}{ Harbour Seal } & Fish Prey & 351 & 1303 & 0 & 2421 & 361 & 449 & 4885 \\
\hline & Invertebrate Prey & 94 & 353 & 0 & 656 & 98 & 121 & 1322 \\
\hline & Total Prey & 445 & 1656 & 0 & 3077 & 459 & 570 & 6207 \\
\hline \multirow[t]{3}{*}{ Totals } & Fish Prey & 2144150 & 661958 & 32294 & 160556 & 40791 & 55909 & 3095658 \\
\hline & Invertebrate Prey & 836626 & 58522 & 4656 & 7209 & 1343 & 1830 & 910186 \\
\hline & Total Prey & 2980776 & 720480 & 36950 & 167765 & 42134 & 57739 & 4005844 \\
\hline
\end{tabular}

TABLE 7. Percentage distribution, within each NAFO area, of major prey type consumption (tons) in Atlantic Canada in 1996, for four species of seals.

\begin{tabular}{|c|c|c|c|c|c|c|c|c|}
\hline \multirow[b]{2}{*}{ Seal Species } & & \multicolumn{7}{|c|}{ NAFO Area } \\
\hline & & $2 \mathrm{~J} 3 \mathrm{KL}$ & $4 \mathrm{RS}$ & $3 \mathrm{M}$ & $4 \mathrm{VsW}$ & $4 \mathrm{~T}$ & Other & All Areas \\
\hline \multirow[t]{3}{*}{ Harp Seal } & Fish Prey & 61.2 & 83.6 & 0.0 & 0.0 & 0.0 & 0.0 & 60.6 \\
\hline & Invertebrate Prey & 26.6 & 8.0 & 0.0 & 0.0 & 0.0 & 0.0 & 21.2 \\
\hline & Total Prey & 87.8 & 91.6 & 0.0 & 0.0 & 0.0 & 0.0 & 81.8 \\
\hline \multirow[t]{3}{*}{ Hooded Seal } & Fish Prey & 10.7 & 1.2 & 87.4 & 0.0 & 0.0 & 0.0 & 9.0 \\
\hline & Invertebrate Prey & 1.5 & 0.1 & 12.6 & 0.0 & 0.0 & 0.0 & 1.2 \\
\hline & Total Prey & 12.2 & 1.2 & 100.0 & 0.0 & 0.0 & 0.0 & 10.2 \\
\hline \multirow[t]{3}{*}{ Grey Seal } & Fish Prey & 0.0 & 7.0 & 0.0 & 94.3 & 96.0 & 96.1 & 7.6 \\
\hline & Invertebrate Prey & 0.0 & 0.0 & 0.0 & 3.9 & 3.0 & 3.0 & 0.2 \\
\hline & Total Prey & 0.0 & 7.0 & 0.0 & 98.2 & 98.9 & 99.0 & 7.8 \\
\hline \multirow[t]{3}{*}{ Harbour Seal } & Fish Prey & 0.0 & 0.2 & 0.0 & 1.4 & 0.9 & 0.8 & 0.1 \\
\hline & Invertebrate Prey & 0.0 & 0.0 & 0.0 & 0.4 & 0.2 & 0.2 & 0.0 \\
\hline & Total Prey & 0.0 & 0.2 & 0.0 & 1.8 & 1.1 & 1.0 & 0.2 \\
\hline \multirow[t]{3}{*}{ Totals } & Fish Prey & 71.9 & 91.9 & 87.4 & 95.7 & 96.8 & 96.8 & 77.3 \\
\hline & Invertebrate Prey & 28.1 & 8.1 & 12.6 & 4.3 & 3.2 & 3.2 & 22.7 \\
\hline & Total Prey & 100.0 & 100.0 & 100.0 & 100.0 & 100.0 & 100.0 & 100.0 \\
\hline
\end{tabular}

$(62 \%)$ in the region (Table 9$)$. On the Flemish Cap (Div. 3M), hooded seals accounted for all of the seal predation. In the northern Gulf (Div. 4RS), the four seal species are present at different times of the year; harp seals are the most abundant, followed by grey, hooded and harbour seals. In this region, harp seals were the most important pinniped predator of redfish $(98 \%)$, capelin $(97 \%)$, sand lance (95\%), Atlantic cod $(75 \%)$ and Atlantic herring (Clupea harengus) (64\%) (Table 9). Grey seals in Div. 4RS were an important predator of Atlantic herring (29\%) and Atlantic cod (25\%), while hooded 
TABLE 8. Estimated consumption (tons), by NAFO area, of some important prey items consumed by four species of seals in 1996.

\begin{tabular}{|c|c|c|c|c|c|c|c|c|}
\hline \multirow{2}{*}{$\begin{array}{l}\text { Seal } \\
\text { Species }\end{array}$} & \multirow{2}{*}{$\begin{array}{c}\text { Prey } \\
\text { Species }\end{array}$} & \multicolumn{7}{|c|}{ NAFO Area } \\
\hline & & $2 \mathrm{~J} 3 \mathrm{KL}$ & $4 \mathrm{RS}$ & $3 \mathrm{M}$ & $4 \mathrm{VsW}$ & $4 \mathrm{~T}$ & Other & All Areas \\
\hline \multirow[t]{8}{*}{ Harp Seal } & Capelin & 708648 & 314412 & & & & & 1023060 \\
\hline & Arctic cod & 175843 & 990 & & & & & 176833 \\
\hline & Sand lance & 345429 & 5871 & & & & & 351300 \\
\hline & G. halibut & 72996 & & & & & & 72996 \\
\hline & Atl. Cod & 48835 & 42089 & & & & & 90924 \\
\hline & Atl. Herring & 30980 & 17878 & & & & & 48858 \\
\hline & Redfish & 16657 & 95128 & & & & & 111785 \\
\hline & All Species & 1399388 & 476368 & & & & & 1875756 \\
\hline \multirow[t]{7}{*}{ Hooded Seal } & Capelin & 3063 & 27 & 333 & & & & 3423 \\
\hline & $\begin{array}{l}\text { Arctic cod } \\
\text { Sand lance }\end{array}$ & 4921 & 1296 & & & & & 6217 \\
\hline & G. halibut & 118273 & 3773 & 11677 & & & & 133723 \\
\hline & Atl. Cod & 33633 & 107 & 3732 & & & & 37472 \\
\hline & Atl. Herring & 4751 & 1252 & & & & & 6003 \\
\hline & Redfish & 17847 & 1842 & 1219 & & & & 20908 \\
\hline & All Species & 182488 & 8297 & 16961 & & & & 207746 \\
\hline \multirow[t]{7}{*}{ Grey Seal } & Capelin & & 9004 & & & 799 & 1097 & 10900 \\
\hline & $\begin{array}{l}\text { Arctic cod } \\
\text { Sand lance }\end{array}$ & & 310 & & 88892 & 18662 & 25600 & 133464 \\
\hline & G. halibut & & 140 & & & 15 & 21 & 176 \\
\hline & Atl. Cod & & 13803 & & 24074 & 7207 & 9887 & 54971 \\
\hline & Atl. Herring & & 8260 & & 12160 & 2870 & 3938 & 27228 \\
\hline & Redfish & & 24 & & 1151 & 250 & 343 & 1768 \\
\hline & All Species & & 31541 & & 126277 & 29803 & 40886 & 228507 \\
\hline \multirow[t]{7}{*}{ Harbour Seal } & Capelin & 26 & 96 & & 179 & 27 & 33 & 361 \\
\hline & Arctic cod & & & & & & & \\
\hline & $\begin{array}{l}\text { Sand lance } \\
\text { G. halibut }\end{array}$ & & & & & & & \\
\hline & Atl. Cod & 27 & 100 & & 185 & 28 & 34 & 374 \\
\hline & Atl. Herring & 115 & 427 & & 793 & 118 & 146 & 1599 \\
\hline & Redfish & 2 & 7 & & 13 & 2 & 2 & 26 \\
\hline & All Species & 170 & 630 & & 1170 & 175 & 215 & 2360 \\
\hline \multirow[t]{8}{*}{ Totals } & Capelin & 711737 & 323539 & 333 & 179 & 826 & 1130 & 1037744 \\
\hline & Arctic cod & 180764 & 2286 & 0 & 0 & 0 & 0 & 183050 \\
\hline & Sand lance & 345429 & 6181 & 0 & 88892 & 18662 & 25600 & 484764 \\
\hline & G. halibut & 191269 & 3913 & 11677 & 0 & 15 & 21 & 206895 \\
\hline & Atl. Cod & 82495 & 56099 & 3732 & 24259 & 7235 & 9921 & 183741 \\
\hline & Atl. Herring & 35846 & 27817 & 0 & 12953 & 2988 & 4084 & 83688 \\
\hline & Redfish & 34506 & 97001 & 1219 & 1164 & 252 & 345 & 134487 \\
\hline & All Species & 1582046 & 516836 & 16961 & 127447 & 29978 & 41101 & 2314369 \\
\hline
\end{tabular}

seals accounted for nearly all (96\%) of the consumption of Greenland halibut by seals in this region. On the eastern Scotian Shelf (Div. 4VsW) and in the southern Gulf (Div. 4T), grey seals were the dominant pinniped predator accounting for virtually all (99\%) of the prey consumption by seals in these two areas.
Most of the consumption of Atlantic cod, Atlantic herring and redfish by seals was comprised of small, juvenile fish (Table 10). In 1996, 95\% of the Atlantic cod consumed by seals were less than $40 \mathrm{~cm}$ in length, $90 \%$ of the herring consumed were less than $30 \mathrm{~cm}$, and $89 \%$ of the redfish consumed were less than $25 \mathrm{~cm}$ in length. 
TABLE 9. Estimated percentage consumption of principal prey species, within each NAFO area, by four species of seals.

\begin{tabular}{|c|c|c|c|c|c|c|c|c|}
\hline \multirow{2}{*}{$\begin{array}{l}\text { Seal } \\
\text { Species }\end{array}$} & \multirow{2}{*}{$\begin{array}{c}\text { Prey } \\
\text { Species }\end{array}$} & \multicolumn{7}{|c|}{ NAFO Area } \\
\hline & & $2 \mathrm{~J} 3 \mathrm{KL}$ & $4 \mathrm{RS}$ & $3 \mathrm{M}$ & $4 \mathrm{VsW}$ & $4 \mathrm{~T}$ & Other & All Areas \\
\hline \multirow[t]{8}{*}{ Harp Seal } & Capelin & 99.57 & 97.18 & & & & & 98.59 \\
\hline & Arctic Cod & 97.28 & 43.31 & & & & & 96.60 \\
\hline & Sand lance & 100.00 & 94.98 & & & & & 72.47 \\
\hline & G. halibut & 38.16 & 0.00 & & & & & 35.28 \\
\hline & Atl. Cod & 59.20 & 75.03 & & & & & 49.48 \\
\hline & Atl. Herring & 86.43 & 64.27 & & & & & 58.38 \\
\hline & Redfish & 48.27 & 98.07 & & & & & 83.12 \\
\hline & All Species & 88.45 & 92.17 & & & & & 81.05 \\
\hline \multirow[t]{8}{*}{ Hooded Seal } & Capelin & 0.43 & 0.01 & 100.00 & & & & 0.33 \\
\hline & Arctic Cod & 2.72 & 56.69 & & & & & 3.40 \\
\hline & Sand lance & 0.00 & 0.00 & & & & & \\
\hline & G. halibut & 61.84 & 96.42 & 100.00 & & & & 64.63 \\
\hline & Atl. Cod & 40.77 & 0.19 & 100.00 & & & & 20.39 \\
\hline & Atl. Herring & 13.25 & 4.50 & & & & & 7.17 \\
\hline & Redfish & 51.72 & 1.90 & 100.00 & & & & 15.55 \\
\hline & All Species & 11.53 & 1.61 & 100.00 & & & & 8.98 \\
\hline \multirow[t]{7}{*}{ Grey Seal } & $\begin{array}{l}\text { Capelin } \\
\text { Arctic Cod }\end{array}$ & & 2.78 & & & 96.73 & 97.08 & 1.05 \\
\hline & Sand lance & & 5.02 & & 100.00 & 100.00 & 100.00 & 27.53 \\
\hline & G. halibut & & 3.58 & & & 100.00 & 100.00 & 0.09 \\
\hline & Atl. Cod & & 24.60 & & 99.24 & 99.61 & 99.66 & 29.92 \\
\hline & Atl. Herring & & 29.69 & & 93.88 & 96.05 & 96.43 & 32.54 \\
\hline & Redfish & & 0.02 & & 98.88 & 99.21 & 99.42 & 1.31 \\
\hline & All Species & & 6.10 & & 99.08 & 99.42 & 99.48 & 9.87 \\
\hline \multirow[t]{8}{*}{ Harbour Seal } & Capelin & & 0.03 & & 100.00 & 3.27 & 2.92 & 0.03 \\
\hline & Arctic Cod & & & & & & & \\
\hline & Sand lance & & & & & & & \\
\hline & G. halibut & & & & & & & \\
\hline & Atl. Cod & 0.03 & 0.18 & & 0.76 & 0.39 & 0.34 & 0.20 \\
\hline & Atl. Herring & 0.32 & 1.54 & & 6.12 & 3.95 & 3.57 & 1.91 \\
\hline & Redfish & 0.01 & 0.01 & & 1.12 & 0.79 & 0.58 & 0.02 \\
\hline & All Species & 0.01 & 0.12 & & 0.92 & 0.58 & 0.52 & 0.10 \\
\hline
\end{tabular}

\section{Discussion}

During model development, several assumptions were made, which may or may not have been realistic. We did not assess the sensitivity of our consumption estimates to the model parameters used in deriving these values - since we used the same model structure as in previous seal feeding and consumption studies (Hammill and Mohn, MS 1994; Mohn and Bowen, 1996; Stenson et al., 1997a). These earlier studies found that pinniped population size was the most important factor affecting fish consumption estimates (Hammill and Mohn, MS 1994; Stenson et al., 1997a). Typically, the annual net rate of increase of seal populations is relatively low (3-10\% per year) and reliable es- timates of population size are available for most of the pinniped species in eastern Canada.

We assumed that average daily age specific energy requirements of seals could be described by a simple equation, where energy intake is a function of body mass ${ }^{0.75}$ multiplied by constants to account for energy requirements due to activity and growth. We did not vary these constants with season, age- or sex-class or diet composition. Although changes in activity and growth do occur, these are poorly documented. Nevertheless, such changes can have a major impact on consumption estimates (Mohn and Bowen, 1996). Age-body mass relationships in our study were based on samples collected after reproduction (when body mass is near the 
TABLE 10. Consumption (tons) of Atlantic cod, Atlantic herring and redfish by seals in 1996 in the northern Gulf of St. Lawrence (NAFO Divs. 4RS), by size group of prey.

\begin{tabular}{|c|c|c|c|c|c|c|}
\hline \multirow[b]{3}{*}{ Seal Species } & \multicolumn{6}{|c|}{ Atlantic cod } \\
\hline & \multicolumn{2}{|c|}{$<40 \mathrm{~cm}$} & \multicolumn{2}{|c|}{$>40 \mathrm{~cm}$} & \multicolumn{2}{|c|}{ Total } \\
\hline & tons & $\%$ of Total & tons & $\%$ of Total & tons & $\%$ of Total \\
\hline $\begin{array}{l}\text { Harp Seal } \\
\text { Hooded Seal }\end{array}$ & 41542 & 98.7 & 547 & 1.3 & 42089 & 75.2 \\
\hline Grey Seal & 11733 & 85.0 & 2070 & 15.0 & 13803 & 24.7 \\
\hline Harbour Seal & 99 & 99.0 & 1 & 1.0 & 100 & 0.2 \\
\hline Total & 53374 & 95.3 & 2618 & 4.7 & 55992 & 100.0 \\
\hline
\end{tabular}

\begin{tabular}{|c|c|c|c|c|c|c|}
\hline \multirow[b]{3}{*}{ Seal Species } & \multicolumn{6}{|c|}{ Atlantic herring } \\
\hline & \multicolumn{2}{|c|}{$<30 \mathrm{~cm}$} & \multicolumn{2}{|c|}{$>30 \mathrm{~cm}$} & \multicolumn{2}{|c|}{ Total } \\
\hline & tons & $\%$ of Total & tons & $\%$ of Total & tons & $\%$ of Total \\
\hline Harp Seal & 16984 & 95.0 & 894 & 5.0 & 17878 & 64.3 \\
\hline Hooded Seal & 901 & 72.0 & 351 & 28.0 & 1252 & 4.5 \\
\hline Grey Seal & 6938 & 84.0 & 1322 & 16.0 & 8260 & 29.7 \\
\hline Harbour Seal & 268 & 62.9 & 158 & 37.1 & 426 & 1.5 \\
\hline Total & 25091 & 90.2 & 2725 & 9.8 & 27816 & 100.0 \\
\hline
\end{tabular}

\begin{tabular}{|c|c|c|c|c|c|c|}
\hline \multirow[b]{3}{*}{ Seal Species } & \multicolumn{6}{|c|}{ Redfish } \\
\hline & \multicolumn{2}{|c|}{$<25 \mathrm{~cm}$} & \multicolumn{2}{|c|}{$>25 \mathrm{~cm}$} & \multicolumn{2}{|c|}{ Total } \\
\hline & tons & $\%$ of Total & tons & $\%$ of Total & tons & $\%$ of Total \\
\hline Harp Seal & 85616 & 90.0 & 9512 & 10.0 & 95128 & 98.1 \\
\hline Hooded Seal & 1105 & 60.0 & 737 & 40.0 & 1842 & 1.9 \\
\hline Grey Seal & 14 & 58.3 & 10 & 41.7 & 24 & $<0.1$ \\
\hline Harbour Seal & \multicolumn{6}{|c|}{ Redfish size frequency data not available } \\
\hline Total & 86735 & 89.4 & 10259 & 10.6 & 96994 & 100.0 \\
\hline
\end{tabular}

annual minimum) and thus underestimate total annual energy requirements. Our approach did not take into account seasonal changes in energy requirements associated with reproduction and moult. However, these energetic costs are unlikely to have a major impact on the annual estimates of energy requirements as Olesiuk (1993) estimated that the additional energy costs associated with reproduction only add about $5 \%$ to the total energy requirements of a population. Also, much of the costs associated with reproduction are offset by a reduction in energy requirements during moulting (Ryg and Øritsland, 1991). However, our assumption that consumption rates do not change during lactation, breeding and moulting periods (when most adults reduce caloric intake and rely on fat reserves to meet their energetic needs) could lead to overestimating consumption if more limited geographical areas or particular seasons were being considered.
There is also some evidence of different seasonal distributions between males and females, as well as sex-specific changes in body condition. For example, after breeding, female harp seals leave the whelping area and feed intensively (Sergeant, 1991; Stenson and Hammill, Northwest Atlantic Fisheries Centre, St. John's, Canada, unpubl. data) to replace a portion of the energy reserves expended during lactation (Beck et al., 1993a, b; Chabot et al., 1996). Males remain near the whelping patch (Sergeant, 1991; Stenson and Hammill, Northwest Atlantic Fisheries Centre, St. John's, Canada, unpubl. data), and continue to lose mass until early April when the moult begins (Chabot et al., 1996).

Consumption estimates of individual fish species are strongly affected by the seasonal distribution patterns of the various seal species, and spatial/ temporal variations in pinniped diet composition. 
Quantitative information on the spatial distribution of seals throughout the year is limited, and large changes in diet composition can occur across years, seasons and geographical areas (Mohn and Bowen, 1996; Shelton et al., 1997). Unfortunately, apart from the harp seals off eastern Newfoundland, temporal and spatial changes in diet composition are not well documented. Stenson et al. (1997a) compared annual estimates of harp seal consumption of Arctic cod, capelin and Atlantic cod off eastern Newfoundland between 1981 and 1994, using both annual and pooled diet composition data. Large annual changes (e.g. 50\%-200\%) in the consumption of major prey species (such as Arctic cod and capelin) were not tracked very well using a pooled average diet, suggesting that important trends in consumption were being masked. Shelton et al. (1997) also examined the effects of uncertainty in the values of population size, energy requirements, residency time, diet composition and prey energy density used by Stenson et al. (1997a) and found that uncertainty associated with the population estimates had the least effect on the overall uncertainty of consumption estimates, while diet composition had the greatest effect.

Invertebrates comprised an important component (12\%-26\%, by weight) of the diet of harp, hooded and harbour seals (Table 6). For hooded and harbour seals, the main invertebrate prey is squid (Appendix Tables 4 and 8). Squid beaks tend to remain lodged in the stomach rugae of seals, which may lead to an overestimation of their dietary importance (Bigg and Fawcett, 1985). With either fish or squid, diet composition can be reconstructed using otolith or beak size, respectively (corrected for digestive state versus body size relationships). However, for most other invertebrates, diet enumeration depends on counting whole prey items and/or recognizable body parts such as eyes or carapaces. Owing to the lower metabolizable energy of invertebrates - and the difficulty in reconstructing the invertebrate component of the diet - we have probably underestimated the role of invertebrates in seal diets, particularly for harp seals. Hence, total prey consumption has likely been underestimated, while fish consumption has probably been overestimated.

The pinniped species for which the greatest amount of information exists (and for which we have the greatest confidence in consumption estimates) is the harp seal. Good data exist on abundance (Shelton et al., 1996; Stenson et al., 1997a;
Stenson et al., MS 1999) and body size (Chabot et al., 1996). Extensive data are also available on geographical and seasonal variations in the diet of harp seals off Newfoundland (Lawson et al., 1995; Lawson and Stenson, 1995, 1997), although less information exists for harp seals in the Gulf of St Lawrence. The available data indicate that the importance of Atlantic cod in the diet of harp seals did not change during 1990 to 1996, despite the extremely low abundance of the Atlantic cod stock in the Newfoundland region (Div. 2J and 3KL) during this time period (Lilly et al., MS 1999).

The population dynamics and diet of grey seals is also quite well known. Estimates of abundance are available for both the Sable Island and northern Gulf of St. Lawrence grey seal population components (Zwanenberg and Bowen, 1990; Hammill and Mohn, MS 1994; Mohn and Bowen, 1996) and diet composition and consumption estimates exist for both components (Benoit and Bowen, 1990; Murie and Lavigne, 1992; Bowen et al., 1993; Bowen and Harrison, 1994; Proust, 1996). However, dietary data for grey seals in the southern Gulf and 'Other' areas is poor. Even less is known about hooded seals; the available data are limited to a few studies on abundance (Bowen et al., 1987; Stenson et al., 1997b) and feeding in nearshore (Ross, 1993) and offshore (Lawson and Stenson, Northwest Atlantic Fisheries Centre, St. John's, Newfoundland, Canada, unpubl. data) areas of Newfoundland. No information exists on the diet of hooded seals in the Gulf of St. Lawrence. In this latter area, prey composition was assumed to be identical to that of hooded seals in inshore Newfoundland. If this assumption is incorrect, the 'true' estimate of consumption by hooded seals in the Gulf could be significantly different from that calculated.

The species for which the least is known is the harbour seal. Our estimates of abundance and distribution are based on work conducted in the 1970s (Boulva and McLaren, 1979) and only a single diet study exists (Bowen and Harrison, 1996). However, the harbour seal population is so small relative to the other three seal species that changes in assumptions concerning the diet of harbour seals would have little effect on the estimates of total and regional pinniped prey consumption.

Estimates of consumption by grey and harp seals presented in this paper differ from earlier estimates (Hammill and Mohn MS 1994; Stenson et al., 1997a). Our estimates of grey seal consump- 
tion: (a) lack a correction factor for unidentified dietary components; (b) do not include a specific cost for the heat increment of feeding; (c) use a lower non-Sable Island population size than Hammill and Mohn (MS 1994); and (d) include a higher proportion of cod in the diet of the northern Gulf of St. Lawrence grey seal component (Proust, 1996). Also, we used the average diet composition of grey seals in the northern Gulf and Scotian Shelf areas to characterize the diets of grey seals in the southern Gulf of St. Lawrence (Div. 4T) and 'Other' regions.

The present estimates of harp seal consumption differ from those provided by Stenson et al. (1997a) in several ways: (1) we used slightly lower estimates for the incremental costs of growth (GP); (2) we limited consumption to the southern part of the harp seal range (Div. 2J and $3 \mathrm{KL}$ and Div. 4RS); and (3) we used population size estimates that assumed pup mortality was greater than adult mortality and which also accounted for a substantially higher harvest of harp seals in Greenland (Stenson et al., MS 1999). Our estimates of consumption also reflect a major change in the spatial distribution of harp seals within Div. 2J and 3KL. Satellite telemetry results indicate that only $11-14 \%$ of harp seals occur in nearshore regions $(<50 \mathrm{~km})$. Thus, diet composition data for the nearshore area was applied to a smaller fraction of the population than in Stenson et al. (1997a).

Little is known about the factors affecting prey selection by seals. A comparison of harp seal stomach content data collected off Newfoundland with prey abundance data collected from research trawl surveys suggests that harp seals preferentially select capelin (and possibly Arctic cod), but are neutrally selective towards Atlantic cod, American plaice and Greenland halibut (Lawson et al., 1998a). Different species of seals also seem to forage on different size groups of prey. For example, in the northern Gulf of St. Lawrence (Div. 4RS), harp seals appear to consume Atlantic cod that are primarily 10-20 cm long (Lawson and Stenson, 1995a), grey seals seem to prefer cod around 15$30 \mathrm{~cm}$ (Benoit and Bowen, 1990; Bowen et al., 1993), while hooded seals prefer cod $25-35 \mathrm{~cm}$ in length (Ross, MS 1993).

The size range of prey consumed by seals can also shift over time. In the northern Gulf, Benoit and Bowen (1990) reported that $85 \%$ of the Atlantic cod consumed by grey seals were $<40 \mathrm{~cm}$ in length. In 1992, Proust (1996) found that 51\% of the Atlantic cod consumed by grey seals were $>40$ $\mathrm{cm}$ in length. Shifts in the size range of prey consumed have also been noted in other areas. Hammond et al. (1994) reported on seasonal changes in the length-frequency distributions of cod, whiting, saithe, sandeels and American plaice consumed by grey seals off northeastern Scotland, while Tollit et al. (1997) noted inter-annual changes in the size distributions of herring and sprat consumed by harbour seals. Bowen et al. (1993) documented differences in the size distributions of cod consumed by grey seals between inshore and offshore areas on the Scotian Shelf, while Stansbury et al. (MS 1998) and Lilly et al. (MS 1999) observed annual differences in the size range of cod consumed by harp seals in waters off Newfoundland.

Predation is an omnipresent, but ephemeral feature of marine ecosystems. Population removals by predation can exceed fisheries harvests, yet it is often assumed that fishing mortality alone is responsible for variations in fish survival (Bax, 1998). Our estimates of consumption indicate that seals in Atlantic Canada consume large quantities of fish but seal predation is only one of several sources of mortality to fish stocks. Realistically, it will not be possible to assess the relative impact of seal predation on fish stock abundance until other sources of natural mortality are quantified. Multispecies predation models indicate that consumption by fish of other fish can exceed that of marine mammals (Overholtz et al., 1991; Trites et al., 1997). Conceptually, multispecies approaches offer the greatest potential to fully evaluate the mortality impacts of predators, but the data requirements of these approaches are too demanding to be of likely use in the near future. Less data intensive, mass-balance types of models (Trites et al., 1997; Walters et al., 1997) are apt to provide more timely insights into ecosystem structure and the relative importance of various species. These insights, in turn, may stimulate more dynamic and predictive modeling efforts that focus on key species. Such efforts would help place predation by seals within the context of overall natural mortality, and facilitate an accurate and synoptic assessment of the impacts of seals on fish stocks.

\section{Acknowledgements}

We thank V. Lesage and D. Chabot, Department of Fisheries and Oceans, Mont Joli, Quebec; and J. W. Lawson, Department of Fisheries and Oceans, 
St John's, Newfoundland for allowing us to use their unpublished data. We also thank J.-F. Gosselin for help with the graphics.

\section{References}

BAX, N. J. 1998. The significance and prediction of predation in marine fisheries. ICES J. Mar. Sci., 55: 997-1030.

BECK, C. G., M. O. HAMMILL, and T. G. SMITH. 1993a. Seasonal variation in the diet of harp seals (Phoca groenlandica) from the Gulf of St. Lawrence and western Hudson Strait. Can. J. Fish. Aquat. Sci., 50: 1363-1371.

BECK, G. G., T. G. SMITH, and M. O. HAMMILL. 1993b. Evaluation of body condition in the Northwest Atlantic harp seal, Phoca groenlandica. Can. J. Fish. Aquat. Sci., 50: 1372-1381.

BENOIT, D., and W. D. BOWEN. 1990. Summer diet of grey seals (Halichoerus grypus) at Anticosti Island, Gulf of St Lawrence, Canada. In: Population biology of sealworm (Pseudoterranova decipiens) in relation to its intermediate and seal hosts. W. D. Bowen (ed.). Can. Bull. Fish. Aquat. Sci., 222: 227-242.

BIGG, M. A., and I. FAWCETT. 1985. Two biases in diet determination of northern fur seals (Callorhinus ursinus). In: Marine Mammals and Fisheries. J. R. Beddington, R. J. H. Beverton, and D. M. Lavigne (eds.). George Allen \& Unwin, London. p. 284 -291.

BOSKOVIC, R., K. M. KOVACS, and M. O. HAMMILL. 1996. Geographic distribution of mitochondrial DNA haplotypes in grey seals Halichoerus grypus. Can. J. Zool., 74: 1787-1796.

BOULVA, J., and I. A. MCLAREN. 1979. Biology of the harbor seal, Phoca vitulina, in eastern Canada. Bull. Fish. Res. Board Can., 200: 23 p.

BOWEN, W. D. 1997. Role of marine mammals in aquatic ecosystems. Mar. Ecol. Prog. Ser., 158: 267274.

BOWEN, W. D., R. A. MYERS, and K. HAY. 1987. Abundance estimation of a dispersed dynamic population: hooded seal (Cystophora cristata) in the Northwest Atlantic. Can. J. Fish. Aquat. Sci., 44: 282-295.

BOWEN, W. D., and G. D. HARRISON. 1994. Offshore diet of grey seals Halichoerus grypus near Sable Island, Canada. Mar. Ecol. Prog. Ser., 112: 1-11.

1996. Comparison of harbour seal diets in two inshore habitats of Atlantic Canada. Can. J. Zool., 74: 25-135.

BOWEN, W. D., J. W. LAWSON, and B. BECK. 1993. Seasonal and geographical variation in the species composition and size of prey consumed by grey seals (Halichoerus grypus) on the Scotian Shelf. Can. J. Fish. Aquat. Sci., 50: 1768-1778.

CAUGHLEY, G. 1977. Analysis of vertebrate populations. J. Wiley \& Sons, Toronto. 234 p.

CHABOT, D., G. B. STENSON, and N. B. CADIGAN. 1996. Short- and long-term fluctuations in the size and condition of harp seal (Phoca groenlandica) in the Northwest Atlantic. NAFO Sci. Coun. Studies, 26: 15-32.

CHRISTENSEN, V. 1995. A model of trophic interactions in the North Sea in 1981, the year of the stomach. Dana, 11: 1-28.

CHRISTENSEN, V., and D. PAULY. 1993. On steady state modelling of ecosystems. In: Trophic Models of Aquatic Ecosystems. V. Christensen and D. Pauly (eds.). ICLARM Conference Proceedings, No. 26: p. 14-19.

COMEAU, N.-A. 1945. La vie et le sport sur la côte nord du bas St. Laurent et du Golfe. Edition Garneau., Québec. 372 p.

CROXALL, J. P., and P. A. PRINCE. 1982. Calorific content of squid (Mollusca: Cephalopoda). Br. Antarct. Surv. Bull., 55: 27-31.

FRCC. 1999. "Fisheries in Transition". Annual Report of the Fisheries Resource Conservation Council and Conservation Requirements for Atlantic Groundfish Stocks for 1999. Fisheries Resource Conservation Council, FRCC.99.R.4, July 1999, 169 p. + Appendices.

GRIFFITHS, D. 1977. Caloric variation in Crustacea and other animals. J. Anim. Ecol., 46: 593-605.

HAMMILL, M. O. MS 1999. Replacement yields of northwest Atlantic grey seals (Halichoerus grypus). DFO Working Paper 99/11, 16 p. Presented at the National Marine Mammal Peer Review Meeting in Montreal, 1-5 February 1999.

HAMMILL, M. O., and J. F. GOSSELIN. 1995. Reproductive rates, age of maturity and age at first birth in Northwest Atlantic grey seals (Halichoerus grypus). Can. J. Fish. Aquat. Sci., 52: 2757-2761.

HAMMILL, M. O., and B. MOHN. MS 1994. A model of grey seal predation on Atlantic cod on the Scotian Shelf and Gulf of St. Lawrence. DFO Atl. Fish. Res. Doc., No. 94/75, 25 p.

HAMMILL, M. O., M. C. S. KINGSLEY, G. BECK, and T. G. SMITH. 1995. Growth and condition in the Northwest Atlantic harp seal. Can. J. Fish. Aquat. Sci., 52: 478-488.

HAMMILL, M. O., C. LYDERSEN, K. M. KOVACS, and B. SJARE. 1997. Estimated fish consumption by hooded seals (Cystophora cristata) in the Gulf of St. Lawrence. J. Northw. Atl. Fish. Sci., 22: 249-257.

HAMMILL, M. O., G. B. STENSON, and R. A. MYERS. 1992. Hooded seal (Cystophora cristata) pup production in the Gulf of St Lawrence. Can. J. Fish. Aquat. Sci., 49: 2546-2550.

HAMMILL, M. O., G. B. STENSON, R. A. MYERS, and W. T. STOBO. 1998. Pup production and population trends of the grey seal (Halichoerus grypus) in the Gulf of St. Lawrence, Canada. Can. J. Fish. Aquat. Sci., 55: 423-430.

HAMMOND, P. S., A. J. HALL, and J. H. PRIME. 1994. The diet of grey seals around Orkney and other island and mainland sites in north-eastern Scotland. J. Appl. Ecol., 31: 340-350. 
HARWOOD, J. 1992. Assessing the competitive effects of marine mammal predation on commercial fisheries. So. Afr. J. Mar. Sci., 12: 689-693.

HARWOOD, J., and J. P. CROXALL. 1988. The assessment of competition between seals and commercial fisheries in the North Sea and the Antarctic. Mar. Mamm. Sci., 4: 13-33.

HISLOP, J. R. G., M. P. HARRIS, and J. G. M. SMITH. 1991. Variation in the calorific value and total energy content of the lesser sandeel (Ammodytes marinus) and other fish preyed on by seabirds. $J$. Zool. (Lond.), 224: 501-517.

HODDER, V. M., L. S. PARSONS, G. H. WINTERS, and K. SPENCER. 1973. Fat and water content of herring in Newfoundland and adjacent waters, 1966-71. Report No. 365. Fish. Res. Board Can., Ottawa, Ont.

KAPEL, F. O., and A. ROSING-ASVID. 1996. Seal hunting statistics for Greenland 1993 and 1994, according to a new system of collecting information, compared to the previous Lists-of-Game. NAFO Sci. Coun. Studies, 26: 71-86.

KLEIBER, M. 1975. The Fire of Life: An Introduction to Animal Energetics. (2nd Ed.) Robert E. Kreiger Publishing Co., New York, 478 p.

LAWSON, J. W., J. T. ANDERSON, E. L. DALLEY, and G. B. STENSON. 1998a. Selective foraging by harp seals, Phoca groenlandica, in nearshore and offshore waters of Newfoundland, 1993 and 1994. Mar. Ecol. Prog. Ser., 163: 1-10.

LAWSON, J. W., and G. B. STENSON. 1995. Historic variation in the diet of harp seals (Phoca groenlandica) in the Northwest Atlantic. In: Whales, seals, fish and man. A. S. Blix, Ø. Ulltang, and L. Walløe (eds.). Elsevier Science B.V., p. 261-269.

1997. The diet of Northwest Atlantic harp seals (Phoca groenlandica) in offshore areas. Can. J. Zool., 75: 2095-2106.

LAWSON, J. W., G. B. STENSON, and D. G. MCKINNON. 1995. Diet of harp seals (Phoca groenlandica) in nearshore waters of the Northwest Atlantic during 1990-1993. Can J. Zool., 73: 18051818 .

LAWSON, J. W., A. M. MAGALHAES, and E. H. MILLER. 1998b. Important prey species of marine vertebrate predators in the Northwest Atlantic: proximate composition and energy density. Mar. Ecol. Prog. Ser., 164: 13-20.

LESLIE, P. H. 1945. On the use of matrices in certain population mathematics. Biometrika, 35: 213-245.

Lilly, G. R., P. A. SHELTON, J. BRATTEY, N. G. CADIGAN, E. F. MURPHY, and D. E. STANSBURY. MS 1999. An assessment of the cod stock in NAFO Divisions 2J+3KL. NAFO SCR Doc., No. 28, Serial No. N4084, 141 p.

MANSFIELD, A.W. 1967. Seals of arctic and eastern Canada. Fish. Res. Bd. Can. Bull., 137.

MARTENSSON, P.-E., A. R. L. GOTAAS, E. S. NORDOY, and A. S. BLIX. 1996. Seasonal change in energy density of prey of northeast Atlantic seals and whales. Mar. Mammal. Sci., 12: 636-640.

MERCER, M. C. (ed.). 1982. Multispecies approaches to fisheries management advice. Can. Spec. Publ. Fish. Aquat. Sci., 59: 169 p.

MOHN, R., and W. D. BOWEN. 1996. Grey seal predation on the Eastern Scotian Shelf: modeling the impact on Atlantic cod. Can. J. Fish. Aquat. Sci., 53: 2722-2738.

MONTEVECCHI, W. A., and J. PIATT. 1984. Composition and energy contents of mature inshore spawning capelin (Mallotus villosus): implications for seabird predators. Comp. Biochem. Physiol. A, 78: 15-20.

MURIE, D. J., and D. M. LAVIGNE. 1991. Food consumption of winter harp seals, Phoca groenlandica, in the St. Lawrence estuary, Canada. Can. J. Zool., 69: $1289-1296$.

1992. Growth and feeding habits of grey seals (Halichoerus grypus) in the northwestern Gulf of St. Lawrence, Canada. Can. J. Zool., 70: 1604-1613.

MYERS, R. A., and G. B. STENSON. 1996. Replacement yield of hooded seals in the Northwest Atlantic. NAFO Sci. Coun. Studies, 26: 121-122.

OLESIUK, P. F. 1993. Annual prey consumption by harbour seals (Phoca vitulina) in the Strait of Georgia, British Columbia. Fisheries Bulletin (Dublin), 91: 491-515.

OVERHOLTZ, W. J., S. A. MURAWSKI, and K. L. FOSTER. 1991. Impact of predatory fish, marine mammals, and seabirds on the pelagic fish ecosystem of the northeastern USA. ICES Mar. Sci. Symp., 193: 198-208.

PERCY, J. A., and F. J. FIFE. 1981. The biochemical composition and energy content of Arctic marine macrozooplankton. Arctic, 34: 307-313.

PROUST, F. 1996. Composition du régime alimentaire du phoque gris (Halichoerus grypus) dans le Golfe du Saint-Laurent, Québec, Canada. Université du Québec à Rimouski, Rimouski, Quèbec, Canada. 72 p.

RONALD, K., K. M. KIEVER, F. W. BEAMISH, and R. FRANK. 1984. Energy requirements for maintenance and faecal and urinary losses of the grey seal (Halichoerus grypus). Can. J. Zool., 62: 1101-1105.

ROSS, S. MS 1993. Food and feeding of the hooded seal in Newfoundland. MSc. Thesis. Memorial University, St. John's, Newfoundland.

RYG, M., and N. A. ØRITSLAND. 1991. Estimates of the energy expenditure and energy consumption of ringed seals (Phoca hispida) throughout the year. Polar Res., 10: 595-601.

SAS INSTITUTE. 1987. SAS/STAT ${ }^{\mathrm{TM}}$ Guide for Personal Computers, Version 6 Edition. Cary, North Carolina, $1028 \mathrm{p}$.

SERGEANT, D. E. 1976. History and present status of populations of harp and hooded seals. Biol. Conserv., 10: 95-118.

SERGEANT, D. E. 1991. Harp seals, man and ice. Can. Spec. Publ. Fish. Aquat. Sci., 114: 153 p.

SHELTON, P. A., G. B. STENSON, B. SJARE, and W. G. WARREN. 1996. Model estimates of harp seal num- 
bers at age for the Northwest Atlantic. NAFO Sci. Coun. Studies, 26: 1-14.

SPARRE, P. 1991. Introduction to multispecies virtual population analysis. ICES Mar. Sci. Symp., 193: 12-21.

STANSBURY, D. E., P. A. SHELTON, G. B. STENSON, B. SJARE, and G. R. LILLY. MS 1998. Catch at age of $2 \mathrm{~J} 3 \mathrm{KL}$ cod in the diet of harp seals. DFO Atl. Fish. Res. Doc., No. 75, 25 p.

STEIMLE, F. W., JR., and R. J. TERRANOVA. 1985. Energy equivalents of marine organisms from the continental shelf of the temperate Northwest Atlantic. J. Northw. Atl. Fish. Sci., 6: 117-124.

STENSON, G. B., R. A. MYERS, M. O. HAMMILL, I.H. NI, W. G. WARREN, and M. C. S. KINGSLEY. 1993. Pup production of harp seals, Phoca groenlandica, in the Northwest Atlantic. Can. J. Fish. Aquat. Sci., 50: 2429-2439.

STENSON, G. B., M. O. HAMMILL, M. C. S. KINGSLEY, B. SJARE, W. G. WARREN, and R. A. MYERS. 1996. 1994 pup production of northwest Atlantic harp seals, Phoca groenlandica. NAFO Sci. Coun. Studies, 26: 47-62.

STENSON, G. B., M. O. HAMMILL, and J. W. LAWSON. 1997a. Predation by harp seals in Atlantic Canada: preliminary consumption estimates for Arctic cod, capelin and Atlantic cod. J.Northw. Atl. Fish. Sci., 22: 137-154.

STENSON, G. B., R. A. MYERS, I-H. NI, and W. G. WARREN. 1997b. Pup production and population growth of hooded seals (Cystophora cristata) near Newfoundland, Canada. Can. J. Fish. Aquat. Sci., 54 (Suppl. 1): 209-216.

STENSON, G. B., B. HEALEY, P. A. SHELTON, and B. SJARE. MS 1999. Recent trends in the population of Northwest Atlantic harp seals, Phoca groenlandica. NAFO SCR Doc., No. 26, Serial No. N4082, 18 p.

STENSON, G. B., and B. SJARE. MS 1997. Seasonal distribution of harp seals, Phoca groenlandica, in the
Northwest Atlantic. ICES C.M. Doc., No. CC:10, $23 \mathrm{p}$.

STOBO, W. T., and G. M. FOWLER. 1994. Aerial surveys of seals in the Bay of Fundy and off southwest Nova Scotia. Can. Tech. Rep. Fish. Aquat. Sci., No. 1943: $57 \mathrm{p}$

STOBO, W. T., and K. C. T. ZWANENBERG. 1990. Grey seal (Halichoerus grypus) pup production on Sable Island and estimates of recent production in the Northwest Atlantic. In: Population biology of sealworm (Pseudoterranova decipiens) in relation to its intermediate and seal hosts. W. D. Bowen (ed.). Can. Bull. Fish. Aquat. Sci., 222: 171-184.

TOLLIT, D. J., S. P. R. GREENSTREET, and P. M. THOMPSON. 1997. Prey selection by harbour seals, Phoca vitulina, in relation to variations in prey abundance. Can. J. Zool., 75: 1508-1518.

TRITES, A. W., V. CHRISTENSEN, and D. PAULY. 1997. Competition between fisheries and marine mammals for prey and primary production in the Pacific Ocean. J. Northw. Atl. Fish. Sci., 22: 173-187.

TYLER, A. V. 1973. Caloric values of some North Atlantic invertebrates. Mar. Biol., 19: 258-261.

WALTERS, C., V. CHRISTENSEN, and D. PAULY. 1997. Structuring dynamic models of exploited ecosystems from trophic mass-balance assessments. Rev. Fish. Biol. Fish., 7: 139-172.

WORTHY, G. A. J. 1990. Nutritional energetics for marine mammals: Addendums. In: CRC handbook of marine mammal medicine: health, disease and rehabilitation. L. A. Dierauf (ed.). CRC Press, Boca Raton, Florida, p. 489-520.

ZWANENBERG, K. C. T., and W. D. BOWEN. 1990. Population trends of the grey seal (Halichoerus grypus) in eastern Canada. In: Population biology of sealworm (Pseudoterranova decipiens) in relation to its intermediate and seal hosts. W. D. Bowen (ed.). Can. Bull. Fish. Aquat. Sci., 222: 185-197. 


\section{Appendix 1. Description of Biology and Model Parameters Used to Estimate Prey Consumption of Harp, Hooded, Grey, and Harbour Seals}

\section{a) Harp Seals}

Population Dynamics. Harp seals in the Northwest Atlantic whelp on the pack ice off the Newfoundland east coast (the Front) and in the Gulf of St. Lawrence (Sergeant, 1991). Total pup production increased from 578000 animals in 1990 to 702900 animals in 1994 (Stenson et al., 1993, 1996). Changes in population size were based on the model used by Stenson et al. (MS 1999). The population model - derived from that of Shelton $e t$ al. (1996) - was updated by incorporating revised estimates of harp seal catches in Greenland and additional data on: (a) recent harvests; (b) age composition of the catches; and (c) current reproductive rates. Pup mortality was assumed to be three times the mortality rate of older seals. Unreported catches were assumed to be $5 \%$ for young-of-the-year animals taken off Canada, and $50 \%$ for all other age groups.

Seasonal Distribution. Harp seals are highly migratory; our knowledge of their seasonal distribution (Appendix Table 1) is primarily based on historical catch data, tag returns and, more recently, satellite telemetry. The Gulf and Newfoundland components of the population intermingle during the non-breeding period, summering in the Canadian Arctic and/or West Greenland. During the autumn and early winter, seals from both components move southward along the Labrador coast. Whelping occurs in the Gulf of St. Lawrence or southern Labrador. After whelping, the animals moult before returning north. Annual changes in ice conditions or food availability (Sergeant, 1991) affect the seasonal movements of the population.

Assumptions concerning the seasonal distribution of harp seals in Div. $2 \mathrm{~J}$ and $3 \mathrm{KL}$ were revised from those of Stenson et al. (1997a). Based on the work of Kapel and Rosing-Asvid (1996) and Sergeant (1991), 20\% of all age groups were assumed to remain in the Arctic throughout the year. The residency period in Div. 2J and 3KL was considered to be 21 November to 6 July based on satellite telemetry tracking data (Stenson and Sjare, MS 1997). One-third of the adult population and $20 \%$ of juveniles (ages 1-4) were assumed to enter the Gulf of St. Lawrence on 1 December and remain there until 30 May. A small proportion (5\%) of the seals that migrated southward were assumed to remain in the study area for the entire year, with the proportion in each area that same as for the winter period.

The proportion of energy obtained by harp seals in the offshore and nearshore areas in Div. $2 \mathrm{~J}$ and $3 \mathrm{KL}$ was also modified from that in Stenson et al. (1997a). We assumed that $89 \%$ and $86 \%$ of the energy required in summer and winter periods, respectively, was obtained in offshore areas (as opposed to 55\% in Stenson et al. 1997a). Our estimates were derived by plotting the locations of seals in Div. 2J and 3KL [obtained from the satellite tracking study described in Stenson and Sjare (MS 1997)] and determining the proportion of sites within $30 \mathrm{~km}$ of the coast (Stenson, Northwest Atlantic Fisheries Centre, St. John's, Nfld., Canada, unpubl. data) where the nearshore diet samples were obtained. Increasing the width of the nearshore band to $50 \mathrm{~km}$ did not significantly increase the proportion of seals in the inshore region (Stenson, Northwest Atlantic Fisheries Centre, St. John's, Nfld., Canada, unpubl. data).

Diet. Diet composition of harp seals in the Gulf (Appendix Table 2) was derived by taking the mean proportions of each prey item in the diet samples collected by Murie and Lavigne (1991), Beck et al. (1993b) and Lawson et al. (1995). The dietary data from Lawson et al. (1995) were supplemented by additional samples collected from the west coast of Newfoundland (Stenson and Lawson, Northwest Atlantic Fisheries Centre, St. John's, Nfld., Canada, unpubl. data).

The diet composition of harp seals off Newfoundland was based on material from Lawson and Stenson (1995, 1997), Lawson et al. (1995) and Stenson and McKinnon (Northwest Atlantic Fisheries Centre, St. John's, Nfld., Canada, unpubl. data). Samples were divided geographically (nearshore/offshore) and by season (summer/ winter) (Appendix Table 2). Seasonal diets in the nearshore area were estimated by averaging the proportion of each prey species in the available seasonal samples (from 1982, 1986, 1990-93; see Stenson et al., 1997a; Lilly et al., MS 1999; and Stenson and McKinnon, Northwest Atlantic Fisheries Centre, St. John's, Nfld., Canada, unpubl. data). 
APPENDIX TABLE 1. Percentage of annual energy requirements obtained by harp seals in different areas of the Northwest Atlantic.

\begin{tabular}{lccrr}
\hline \hline & \multicolumn{2}{c}{ Pups/Juveniles } & \multicolumn{2}{c}{ Adults } \\
\cline { 2 - 3 } NAFO Area & $\begin{array}{c}\text { Winter } \\
\text { (Oct-Mar) }\end{array}$ & $\begin{array}{c}\text { Summer } \\
\text { (Apr-Sep) }\end{array}$ & $\begin{array}{c}\text { Winter } \\
\text { (Oct-Mar) }\end{array}$ & $\begin{array}{c}\text { Summer } \\
\text { (Apr-Sep) }\end{array}$ \\
\hline Div. 2J3KL (inshore) & 3.32 & 2.14 & 2.82 & 1.91 \\
Div. 2J3KL (offshore) & 20.41 & 17.31 & 17.35 & 15.58 \\
Div. 4RS (Northern Gulf) & 5.47 & 2.99 & 9.03 & 4.95 \\
SA 0+1 (Arctic) & 20.66 & 27.70 & 20.66 & 27.70 \\
All Areas & 49.86 & 50.14 & 49.86 & 50.14 \\
\hline
\end{tabular}

APPENDIX TABLE 2. Harp seal diet composition data (\% wet weight) used to estimate prey consumption.

\begin{tabular}{|c|c|c|c|c|c|}
\hline \multirow[b]{3}{*}{ Species } & \multicolumn{4}{|c|}{ Newfoundland } & \multirow{3}{*}{$\begin{array}{l}\text { Gulf } \\
\text { All }\end{array}$} \\
\hline & \multicolumn{2}{|c|}{ Nearshore } & \multicolumn{2}{|c|}{ Offshore } & \\
\hline & Summer & Winter & Summer & Winter & \\
\hline Arctic cod & 23.0 & 50.8 & 0.8 & 2.2 & 0.1 \\
\hline Atlantic cod & 5.1 & 3.0 & 2.1 & 1.0 & 6.4 \\
\hline Capelin & 21.1 & 11.2 & 34.7 & 30.9 & 47.7 \\
\hline Atlantic herring & 12.6 & 7.3 & 0.0 & 0.0 & 2.7 \\
\hline Sand lance & 0.5 & 0.2 & 30.5 & 0.0 & 0.9 \\
\hline Redfish & 0.3 & $<0.1$ & 1.1 & 0.3 & 14.4 \\
\hline Greenland halibut & 1.5 & 1.0 & 4.8 & 2.0 & 0.0 \\
\hline American plaice & 1.1 & 0.2 & 0.1 & 1.1 & 0.0 \\
\hline Pleuronectidae & 2.1 & 0.6 & 7.4 & 5.7 & 11.0 \\
\hline Windowpane & 0.0 & 0.0 & 0.0 & 0.0 & 2.0 \\
\hline Atlantic salmon & 0.1 & 0.0 & 0.0 & 0.0 & 0.4 \\
\hline Sculpin & 0.6 & 1.8 & 0.2 & 0.3 & 0.8 \\
\hline Silver hake & 0.0 & 0.0 & 0.0 & 0.0 & 0.5 \\
\hline White hake & 0.0 & 0.3 & 0.4 & 0.1 & 0.1 \\
\hline Smelt & 0.0 & 0.1 & 0.0 & 0.0 & $<0.1$ \\
\hline Alewife & 0.0 & 0.0 & 0.0 & 0.0 & $<0.1$ \\
\hline Haddock & 0.0 & 0.0 & 0.0 & 0.0 & 0.4 \\
\hline Gadus sp. & 0.7 & 1.4 & $<0.1$ & $<0.1$ & 0.5 \\
\hline Other Fish & 11.7 & 9.9 & 0.5 & 14.7 & 3.4 \\
\hline Total Fish & 80.4 & 87.8 & 82.6 & 58.3 & 91.3 \\
\hline Shrimp & 5.5 & 1.3 & 6.8 & 31.2 & 2.0 \\
\hline Squid & 0.9 & 0.7 & 0.2 & 0.6 & 1.0 \\
\hline Euphausiid & 3.8 & 0.6 & $<0.1$ & 0.0 & $<0.1$ \\
\hline Other Invertebrates & 9.4 & 9.6 & 10.4 & 9.9 & 5.7 \\
\hline Total Invertebrates & 19.6 & 12.2 & 17.4 & 41.7 & 8.7 \\
\hline Total Prey & 100.0 & 100.0 & 100.0 & 100.0 & 100.0 \\
\hline $\begin{array}{l}\text { Mean Energy Content } \\
\text { (Kjoules/gram) }\end{array}$ & 6.2 & 5.6 & 6.0 & 6.0 & 6.3 \\
\hline
\end{tabular}




\section{b) Hooded Seals}

Population Dynamics. Hooded seals whelp during March on the pack ice off the Newfoundland east coast (the Front), in the Gulf of St. Lawrence, and in the Davis Strait (Sergeant, 1976). Little is known about stock affinities between the three population groups. In 1984, pup production was estimated to be 62400 animals at the Front and 19 000 animals in Davis Strait (Bowen et al., 1987). Pup production was not determined in the Gulf. In 1990, pup production was estimated to be 83100 at the Front (Stenson et al., 1997b) and 2006 in the Gulf (Hammill et al., 1992). Current estimates of abundance are not available for any of the three whelping patches. An annual population rate of increase of $4.8 \%$ in Newfoundland waters was calculated from the 1984 and 1990 estimates of pup production at the Front, and applied to the Gulf population. Reproductive rates were set at 0.028 , $0.262,0.504,0.654,0.734,0.802,0.850,0.908$, and 0.970 for ages 3 to $11+$ years (Stenson, Northwest Atlantic Fisheries Centre, St. John's, Nfld., Canada, unpubl. data). Adult mortality was set at $9 \%$ per year, intermediate to values of 0.07 and 0.13 used in other studies (Myers and Stenson, 1996; Hammill et al., 1997). Juvenile mortality was initially set at 3 times the adult mortality rate $(3 \times 9 \%=27 \%)$ and then increased to $40 \%$, generating a total net annual population increase of $4.8 \%$.

Seasonal Distribution. Seasonal distributions (Appendix Table 3) were based on: (1) satellite telemetry data obtained after whelping had occurred at the Front and in the Gulf (Stenson and Hammill, Northwest Atlantic Fisheries Centre, St. John's, Nfld., Canada, unpubl. data); (2) observations by fishermen and hunters; and (3) historical accounts (Comeau, 1945). We assumed that all pups and one- year-old seals remained in Arctic waters ( Subareas $0+1)$. As well, we assumed that only $20 \%$ of juveniles aged $2+$ and $3+$ returned each year to the Front (Div. 2J and 3KL) or to the northern Gulf (Div. 4RS). All adults were assumed to annually return to one or the other of these areas.

Diet. Dietary data (Appendix Table 4) were divided into inshore (Ross, MS 1993) and offshore components (Lawson and Stenson, Northwest Atlantic Fisheries Centre, St. John's, Nfld., Canada, unpubl. data). Inshore data from the Front was used to estimate hooded seal consumption in the Gulf.

\section{c) Grey Seals}

Population Dynamics. Most grey seals whelp on either Sable Island or on the Gulf of St Lawrence pack ice. Although the two groups form a single stock (Boskovic et al., 1996), it is convenient to consider seals in the two areas as separate groups. Pup production estimates from the 1980 s are available from both groups (Stobo and Zwanenburg, 1990; Hammill et al., 1998). The population was reconstructed and projected forwards from 1990 to 1996, using age-specific reproductive rates of $0.176,0.860$, and 0.876 for animals aged 4,5 and 6 years, respectively (Hammill and Gosselin, 1995). Adult survival rates were set at 0.96 . To achieve the reported rate of increase in the Sable Island herd (12.6\%; Zwanenburg and Bowen, 1990), juvenile survivorship was set at 0.787 . The Sable Island component thus increased from 62920 animals in 1990 to 128500 animals in 1996 . The dynamics of the non-Sable Island group were different owing to: (1) culling on the whelping patch from 1980 to 1984; (2) a bounty hunt which was discontinued in 1990; and (3) the likelihood of higher pup mortality due to the instability of the pack ice habitat

APPENDIX TABLE 3. Quarterly distribution (\% of total population in each area) of Gulf of St. Lawrence (Gulf Herd) and eastern Newfoundland (Front Herd) hooded seals [see text "Seasonal Distribution" for explanation of the proportions of the herds remaining in the Arctic.]

\begin{tabular}{lrrrrrrrrr}
\hline \hline & \multicolumn{4}{c}{ Gulf Herd } & & \multicolumn{3}{c}{ Front Herd } \\
\cline { 2 - 8 } NAFO Area & Q1 & Q2 & Q3 & Q4 & & Q1 & Q2 & Q3 & Q4 \\
\hline Div. 4RS (Northern Gulf) & 100 & 100 & 0 & 17 & & 0 & 0 & 0 & 0 \\
Div. 2J3KL (Inshore) & 0 & 0 & 0 & 1 & & 10 & 3 & 0 & 3 \\
Div. 2J3KL (Offshore) & 0 & 0 & 0 & 15 & & 90 & 31 & 0 & 30 \\
Div. 3M (Flemish Cap) & 0 & 0 & 0 & 0 & & 0 & 17 & 0 & 0 \\
Arctic (SA 0 + 1) & 0 & 0 & 100 & 67 & & 0 & 49 & 100 & 67 \\
All Areas & 100 & 100 & 100 & 100 & & 100 & 100 & 100 & 100 \\
\hline
\end{tabular}


APPENDIX TABLE 4. Hooded seal diet composition data (\% wet weight) used to estimate prey consumption.

\begin{tabular}{lcc}
\hline \hline & \multicolumn{2}{c}{ NAFO Area } \\
\cline { 2 - 3 } Species & $\begin{array}{c}\text { Gulf (Div. 4RS) \& Div. 2J3KL } \\
\text { (Inshore) }\end{array}$ & $\begin{array}{c}\text { Div. 2J3KL } \\
\text { (Offshore) }\end{array}$ \\
\hline Arctic cod & 14.5 & 0.0 \\
Atlantic cod & 1.2 & 10.1 \\
Capelin & 0.3 & 0.9 \\
Atlantic herring & 14.0 & 0.0 \\
Witch flounder & 0.0 & 15.5 \\
Redfish & 20.6 & 3.3 \\
Greenland halibut & 42.2 & 31.6 \\
Pleuronectidae & 0.0 & 8.3 \\
Grenadier & 0.0 & 5.7 \\
Blue hake & 0.0 & 4.2 \\
Lancetfish & 0.0 & 1.6 \\
Eelpout & 0.0 & 0.4 \\
Other Fish & 0.0 & 5.8 \\
Total Fish & 92.8 & 87.4 \\
Squid & 7.2 & 12.6 \\
Total Prey & 100.0 & 100.0 \\
Mean Energy Content & 5.4 & 5.2 \\
Kjoules/gram) & & \\
\hline
\end{tabular}

(Zwanenburg and Bowen, 1990; Hammill et al., 1998). Mark-recapture studies indicate that pup production of the non-Sable Island component was increasing at 7.4\%, although a 1997 aerial suggested that the increase was only about $3.4 \%$ (Hammill, MS 1999). Assuming no differences in adult survival rates between the Gulf and Sable Island components of the population, we set pup survival at 0.329 to achieve an increase in pup production of $\approx 3.4 \%$ between 1984 and 1996. Using all of the parameters above, the total grey seal population increased from 96900 animals in 1990 to 173500 animals in 1996 (Table 2).

Seasonal Distribution. The seasonal distribution of grey seals (Appendix Table 5) followed that reported in Hammill and Mohn (MS 1994).

Diet. Dietary information on grey seals in the northern Gulf Div. 4RS) and on the eastern Scotian Shelf (Div. 4VsW) is available from several studies (Bowen et al., 1993; Bowen and Harrison, 1994, 1996) (Appendix Table 6). Knowledge of grey seal diets in the southern Gulf and 'Other' areas, however, is based on very limited sampling. For the northern Gulf, all available sampling data (Benoit and Bowen, 1990; Murie and Lavigne, 1992; Proust, 1996) were combined and the average contribution of each prey species was calculated. Dietary data for the Scotian Shelf were divided into summer (April-September) and winter (October-March) components (Bowen et al., 1993; Bowen and Harrison, 1994). Diet composition for the southern Gulf and 'Other' regions was based on an average diet using samples from all areas.

\section{d) Harbour seals}

Population Dynamics. The harbour seal is widely distributed throughout Atlantic Canada; however, there are no reliable estimates of population abundance or trends in stock size. In 1973, the population was estimated at 12700 animals (Boulva and McLaren, 1979) and thought to be declining at $4 \%$ per year due to high bounty takes. In 1976, the bounty program was eliminated. To reconstruct the dynamics of the Atlantic harbour seal population, we assumed that 12700 animals existed in 1973, and that the population declined at 
APPENDIX TABLE 5. Quarterly distribution (\% of total population in each area) of Gulf of St. Lawrence (Gulf Herd) and Sable Island (Sable Herd) grey seals.

\begin{tabular}{|c|c|c|c|c|c|c|c|c|}
\hline \multirow[b]{2}{*}{ NAFO Area } & \multicolumn{4}{|c|}{ Gulf Herd } & \multicolumn{4}{|c|}{ Sable Herd } \\
\hline & Q1 & Q2 & Q3 & Q4 & Q1 & Q2 & Q3 & Q4 \\
\hline Div. 4RS (Northern Gulf) & 10.0 & 35.0 & 60.0 & 50.0 & 0.0 & 12.5 & 7.5 & 5.0 \\
\hline Div. 4T (Southern Gulf) & 70.0 & 35.0 & 10.0 & 30.0 & 0.0 & 12.5 & 7.5 & 0.0 \\
\hline Div. 4VsW (Eastern Scotian Shelf) & 10.0 & 20.0 & 20.0 & 10.0 & 90.0 & 50.0 & 50.0 & 80.0 \\
\hline Other (Div. 4X; Subdiv. 3Ps) & 10.0 & 10.0 & 10.0 & 10.0 & 10.0 & 25.0 & 35.0 & 15.0 \\
\hline All Areas & 100.0 & 100.0 & 100.0 & 100.0 & 100.0 & 100.0 & 100.0 & 100.0 \\
\hline
\end{tabular}

APPENDIX TABLE 6. Grey seal diet composition data (\% wet weight) used to estimate prey consumption.

\begin{tabular}{|c|c|c|c|c|}
\hline \multirow[b]{2}{*}{ Species } & \multicolumn{2}{|c|}{ Eastern Scotian Shelf (Div. 4VsW) } & \multicolumn{2}{|c|}{ Southern Gulf (Div. 4T)\& } \\
\hline & Summer & Winter & Northern Gulf (Div. 4RS) & Other $(4 \mathrm{X} ; 3 \mathrm{Ps})$ \\
\hline Capelin & 0.0 & 0.0 & 6.0 & 1.2 \\
\hline Sand lance & 47.2 & 65.1 & 0.9 & 45.1 \\
\hline Pleuronectidae & 8.1 & 7.0 & 0.0 & 6.0 \\
\hline Greenland halibut & 0.0 & 0.0 & 0.2 & $<0.1$ \\
\hline Atlantic cod & 17.6 & 9.7 & 41.2 & 19.1 \\
\hline Redfish & 0.6 & 0.9 & 0.1 & 0.6 \\
\hline Atlantic herring & 11.0 & 1.5 & 12.5 & 7.5 \\
\hline Witch flounder & 0.0 & 0.0 & 0.1 & 0.0 \\
\hline American plaice & 0.0 & 0.0 & 3.7 & 0.7 \\
\hline Sculpin & 0.0 & 0.0 & 1.1 & 0.2 \\
\hline Mackerel & 0.1 & 7.9 & 5.7 & 4.3 \\
\hline Silver hake & 7.1 & 0.6 & 0.0 & 3.1 \\
\hline Lumpfish & 0.0 & 0.0 & 7.4 & 1.5 \\
\hline Wolffish & 0.0 & 0.0 & 6.0 & 1.2 \\
\hline White hake & 0.0 & 0.0 & 0.3 & 0.1 \\
\hline Pollock & 2.3 & 0.0 & 0.0 & 0.9 \\
\hline Haddock & 0.0 & 0.0 & 1.2 & 0.2 \\
\hline Atlantic salmon & 0.0 & 0.0 & 0.9 & 0.2 \\
\hline Ocean pout & 0.0 & 0.0 & 5.5 & 1.1 \\
\hline Eelpout & 0.0 & 0.0 & 0.1 & $<0.1$ \\
\hline Yellowtail flounder & 0.0 & 0.0 & 3.4 & 0.7 \\
\hline Alewife & 0.0 & 0.0 & 1.1 & 0.2 \\
\hline Skates & 0.0 & 0.0 & 2.2 & 0.4 \\
\hline Winter flounder & 0.0 & 0.0 & 0.2 & 0.0 \\
\hline Cunner & 0.0 & 0.0 & 0.1 & $<0.1$ \\
\hline Other Fish & 1.4 & 4.5 & 0.0 & 2.4 \\
\hline Total Fish & 95.4 & 97.2 & 99.9 & 97.0 \\
\hline Squid & 4.6 & 2.8 & 0.1 & 3.0 \\
\hline Total Prey & 100.0 & 100.0 & 100.0 & 100.0 \\
\hline $\begin{array}{l}\text { Mean Energy Content } \\
\text { (Kjoules/gram) }\end{array}$ & 5.9 & 6.3 & 5.9 & 6.1 \\
\hline
\end{tabular}


APPENDIX TABLE 7. Quarterly distribution (\% of total population in each area) of harbour seals, by area, in Atlantic Canada.

\begin{tabular}{lrrrr}
\hline \hline NAFO Area & Q1 & Q2 & Q3 & Q4 \\
\hline Div. 4RS (Northern Gulf) & 26.4 & 26.4 & 26.4 & 26.4 \\
Div. 4T (Southern Gulf) & 8.0 & 8.0 & 8.0 & 8.0 \\
Div. 2J3KL (Newfoundland) & 7.9 & 7.9 & 7.9 & 7.9 \\
Div. 4VsW (Eastern Scotian Shelf) & 48.0 & 48.0 & 48.0 & 48.0 \\
Other (Div. 4X; Subdiv. 3Ps) & 9.7 & 9.7 & 9.7 & 9.7 \\
All Areas & 100.0 & 100.0 & 100.0 & 100.0 \\
\hline
\end{tabular}

4\% per year until 1976 when the bounty program ceased. As harbour seals appear to be increasing in the Bay of Fundy, around the Nova Scotian coast, and in the St. Lawrence Estuary (Stobo and Fowler, 1994; V. Lesage, Institute Maurice Lamontagne, Mont Joli, Quebec, Canada, unpubl. data), we assumed that the total harbour seal population in eastern Canada has been increasing since 1976. Adult survivorship was therefore set at 0.9 and juvenile mortality rates were assumed to be 3 times the adult mortality rate (i.e., $3 \times 0.1$ ), resulting in a juvenile survival rate of 0.7 . Age specific reproductive rates were set at $0.27,0.55,0.79$ and 0.94 for animals aged $4+, 5+, 6+$ and $>6+$, respectively (Boulva and McLaren, 1979). These parameters resulted in a net rate of increase of $5.6 \%$ per year, and generated an increase in population size from 22800 animals in 1990 to 31900 animals in 1996 (Table 2).

Seasonal Distribution. The geographical distribution of the population was based on the findings of Boulva and McLaren (1979). As satellite telemetry data indicate that harbour seals are relatively sedentary (Lesage and Hammill, Institute Maurice Lamontagne, Mont Joli, Quebec, Canada, unpubl. data), we assumed that there were no seasonal changes in the areal distribution of the population during the year (Appendix Table 7).

Diet. Quantitative diet information for harbour seals is quite limited. Bowen and Harrison (1996) examined the diet composition of harbour seal in the Bay of Fundy and on the Nova Scotia eastern shore. Their dietary findings for harbour seals were applied in all areas for all seasons (Appendix Table 8).

APPENDIX TABLE 8. Harbour seal diet composition data (\% wet weight) used to estimate prey consumption.

\begin{tabular}{lr}
\hline \multicolumn{1}{c}{ Species } & \% Wet Weight \\
\hline Atlantic herring & 24.4 \\
Pollock & 12.7 \\
Atlantic cod & 5.7 \\
Capelin & 5.5 \\
White hake & 2.9 \\
Alewife & 1.7 \\
Mackerel & 1.4 \\
Winter flounder & 1.3 \\
Ocean pout & 0.8 \\
Silver hake & 0.7 \\
Redfish & 0.4 \\
Cunner & 0.3 \\
Sculpin & 0.2 \\
Haddock & 0.1 \\
Blueback herring & 0.1 \\
Butterfish & 1.1 \\
Fourspot flounder & 0.1 \\
Other Fish & 19.1 \\
Total Fish & 78.5 \\
Squid & 14.8 \\
Crab & 0.4 \\
Other Invertebrates & 6.3 \\
Total Invertebrates & 21.5 \\
Total Prey & 100.0 \\
Mean Energy Content (Kjoules/gram) & 5.3 \\
\hline & \\
& \\
&
\end{tabular}

